\title{
MINERAL EQUILIBRIA, GEOTHERMOMETERS AND GEOBAROMETERS IN SOME ICELANDIC HYALOCLASTITES
}

\author{
HEIKKI MÄKIPÄ
}

\begin{abstract}
MÄKIPÄÄ, HEIKKI 1978: Mineral equilibria, geothermometers and geobarometers in some Icelandic hyaloclastites. Bull. Geol. Soc. Finland 50: 113-134.

Distribution ccefficients between crystal and magma can be used to establish various properties of magmas. A number of natural hyaloclastites have been used to evaluate some of the established geothermometers and geobarometers. Comparison with experimental data on the same rocks shows that the olivine geothermometer gives the most reasonable results and in some cases plagioclase geothermometer can also be useful.

However before mineral-liquid combinations can be used to evaluate magma properties equilibrium between the various phases must be established. This is not always simple and more experimental data is needed.
\end{abstract}

Heikki Mäkipää, Nordic Volcanological Institute, University of Iceland, Geoscience Building, 101 Reykjavik, Iceland.

\section{Introduction}

The purpose of this paper is to describe the mineral liquid relationships in some common natural Icelandic hyaloclastites. The rocks used here are described by Mäkipää (1978).

The rocks are basalts quenched in water (mainly glacial melt water) upon eruption. Phenocrysts (olivine, plagioclase and clinopyroxene) are therefore unaffected by reactions occurring upon cooling and crystallization of subaerial lavas. All samples are quartz- or olivine normative tholeiites not far from the normative quartz saturation plane.
The quickly cooled glasses offer the possibility to study the state of equilibrium between crystals and melt. The pressure and temperature conditions of the magma during the crystallization of phenocryst phases prior to eruption can be inferred from existing geothermometers and geobarometers.

The geothermometers and geobarometers used are derived from experimental works where the distribution of elements between the coexisting phases have been derived as a function of temperature, total pressure and the fugacities of important volatile components such as water and oxygen. The experiments of Roeder and Emslie (1970), Kudo and 
Table 1. Microprobe analyses of some selected rep resentative experimental products.

\begin{tabular}{|c|c|c|c|c|c|c|c|c|c|}
\hline \multirow{3}{*}{$\begin{array}{l}\text { Sample No. } \\
\text { Run No. } \\
\text { Temperature }\left({ }^{\circ} \mathrm{C}\right)\end{array}$} & \multicolumn{5}{|c|}{ KRA-731 } & \multicolumn{4}{|c|}{ NAL-13 1} \\
\hline & \# 7313 & \# 7312 & \# 7314 & \# 7315 & \# 7311 & & $\# 3$ & \# 5 & $\# 6$ \\
\hline & 1197 & 1192 & 1186 & 1180 & 1161 & 2 & 1258 & 1202 & 1182 \\
\hline \multicolumn{10}{|l|}{ Liquid } \\
\hline $\mathrm{SiO}_{2}$ & 49.93 & 49.67 & 50.19 & 48.83 & 49.47 & 47.60 & 47.77 & 48.47 & 49.12 \\
\hline $\mathrm{TiO}_{2}$ & 1.70 & 1.65 & 1.68 & 1.73 & 1.84 & 1.09 & 1.21 & 1.69 & 1.82 \\
\hline $\mathrm{Al}_{2} \mathrm{O}_{3}$ & 13.83 & 13.20 & 13.37 & 13.99 & 13.44 & 16.66 & 15.90 & 13.82 & 13.59 \\
\hline $\mathrm{FeO}^{\mathrm{t}}$ & 12.27 & 12.07 & 11.93 & 12.00 & 12.69 & 9.56 & 9.51 & 10.76 & 11.20 \\
\hline $\mathrm{MnO}$ & 0.22 & 0.23 & 0.25 & 0.24 & 0.24 & 0.13 & 0.17 & 0.24 & 0.19 \\
\hline $\mathrm{MgO}$ & 7.23 & 7.07 & 6.93 & 6.88 & 6.58 & 9.56 & 10.22 & 8.75 & 7.59 \\
\hline $\mathrm{CaO}$ & 11.63 & 11.39 & 11.71 & 10.93 & 11.15 & 12.76 & 12.92 & 13.32 & 12.22 \\
\hline $\mathrm{Na}_{2} \mathrm{O}$ & 2.20 & 2.22 & 2.25 & 2.02 & 2.00 & 2.14 & 1.81 & 2.03 & 2.00 \\
\hline $\mathrm{K}_{2} \mathrm{O}$ & 0.26 & 0.25 & 0.24 & 0.27 & 0.26 & 0.11 & 0.13 & 0.16 & 0.14 \\
\hline $\mathrm{P}_{2} \mathrm{O}_{5}$ & 0.11 & 0.16 & 0.17 & 0.18 & 0.20 & 0.09 & 0.09 & 0.19 & 0.17 \\
\hline \multicolumn{10}{|l|}{ Olivine } \\
\hline $\mathrm{SiO}_{2}$ & & 39.08 & 38.56 & 38.55 & 38.47 & & 39.43 & 40.29 & 38.75 \\
\hline $\mathrm{FeO}^{\mathrm{t}}$ & & 19.62 & 20.33 & 20.41 & 22.51 & & 16.13 & 15.28 & 17.11 \\
\hline $\mathrm{MnO}$ & & 0.38 & 0.35 & 0.42 & 0.36 & & 0.30 & 0.24 & 0.26 \\
\hline $\mathrm{MgO}$ & & 39.43 & 39.26 & 39.41 & 38.90 & & 47.60 & 43.47 & 45.17 \\
\hline $\mathrm{CaO}$ & & 0.26 & 0.29 & 0.33 & 0.48 & & 0.47 & 0.37 & 0.41 \\
\hline \multicolumn{10}{|l|}{ Plagioclase } \\
\hline $\mathrm{SiO}_{2}$ & & & & 50.45 & 50.50 & & 46.93 & 48.85 & 47.81 \\
\hline $\mathrm{Al}_{2} \mathrm{O}_{3}$ & & & & 30.80 & 30.27 & & 32.06 & 31.60 & 31.37 \\
\hline $\mathrm{FeO}^{\mathrm{t}}$ & & & & 0.71 & 0.85 & & 0.63 & 0.70 & 0.69 \\
\hline $\mathrm{MgO}$ & & & & - & - & & 0.27 & 0.25 & 0.25 \\
\hline $\mathrm{CaO}$ & & & & 15.14 & 14.86 & & 18.39 & 16.91 & 15.47 \\
\hline $\mathrm{Na}_{2} \mathrm{O}$ & & & & 2.54 & 2.66 & & 1.56 & 2.29 & 2.47 \\
\hline $\mathrm{K}_{2} \mathrm{O}$ & & & & 0.06 & 0.12 & & 0.02 & 0.09 & - \\
\hline $\begin{array}{l}\text { Other phases } \\
\text { present }\end{array}$ & & & & & CPX & & & & CPX \\
\hline
\end{tabular}

1 Experiments by Dr. S. Steinthorsson (unpubl. data)

2 Analysis by Sigvaldason (1974)

Weill (1970) and Drake (1972) are of particular relevance to the present study.

Some of the samples have also been used for experiments and the results are used for comparison with other experimental results.

The question of equilibrium is also relevant and before the temperature and pressure conditions can be derived the existence of equilibrium needs to be established. In the following account no attempt is made to establish various properties of the hyaloclastites at the time of eruption. The results sofar indicate that useful approximations can be obtained.

\section{Melting experiments}

Melting experiments were performed in order to evaluate the possible compositional effect on the distribution coefficients. Some of the experimental results are given in Table 1.

The experiments were carried out in a $\mathrm{Pt}$ wound vertical tube quench furnace. The run temperatures were measured using $\mathrm{Pt} / \mathrm{Pt}$ $10 \% \mathrm{Rh}$ thermocouple before and after each run. The temperature fluctuation during each run was within $1^{\circ} \mathrm{C}$. The total pressure in the runs was the atmospheric pressure and the furnace atmosphere was a mixture of 
carbon dioxide and hydrogen (close to the QMF-buffer) which was passed upwards through the furnace at a constant rate. In some runs the oxygen fugacity was controlled following the QMF-buffer and in some runs the oxygen fugacity was kept constant (constant gas mixture and flow rate).

The glasses which were melted at $1315^{\circ} \mathrm{C}$ were suspended directly into the furnace at the desired temperature. After approximately 24 hours the platinum wire was melted with an electric current so that the sample fell down into the water where it quenched within seconds. The run duration of 24 hours appears to be sufficient for the attaintment of equilibrium (e.g. Kennedy 1948, Yoder and Tilley 1962, Seward 1971, Sun et al. 1974, Duke 1976). However, Drake and Weill (1975) and Watson (1977) used much longer time to equilibrate their samples. Experiments conducted at the same temperature using longer run times gave identical results and in the present study some 3 and 5 day runs were made without a noticeable change. Equilibrium is also indicated by the homogenity of the produced phases in the present study.

\section{Olivine - liquid combination}

\section{General}

Bowen and Schairer (1935) demonstrated that there is a complete solid solution between forsterite and fayalite in the system $\mathrm{MgO}-\mathrm{FeO}-\mathrm{SiO}_{2}$.

This system provides a simple model to determine the composition of the equilibrium olivine crystals. Compositions (listed in Table 2) were read directly from the phase diagram $\mathrm{Mg}_{2} \mathrm{SiO}_{4}-\mathrm{Fe}_{2} \mathrm{SiO}_{4}$.

\section{Equilibrium distribution coefficient}

According to Roeder and Emslie (1970) the distribution of magnesium and ferrous iron between olivine and liquid can be expressed by the following equation:

$$
\begin{aligned}
& \mathrm{Mg}(\text { olivine })+\mathrm{Fe}(\text { liquid })=\mathrm{Mg}(\text { liquid })+ \\
& \mathrm{Fe}(\text { olivine })
\end{aligned}
$$

The replacement of the smaller $\mathrm{Mg}$ by the larger $\mathrm{Fe}$ ion results in a lowering of the melting temperature. An equilibrium constant $(\mathrm{K})$ for this reaction can be written in terms of the activities (a) of the various chemical species, i.e. ( $\mathrm{Mg}, \mathrm{MgO}, \mathrm{MgSi}_{0.5} \mathrm{O}_{2}$ ) in the liquid and solid solutions or in terms of the mole fraction (X) and activity coefficients $(\gamma)$ of the various species:

$$
\begin{aligned}
& \mathrm{K}=\frac{\left(\mathrm{a}_{\mathrm{FeO}}^{\mathrm{ol}}\right)\left(\mathrm{a}_{\mathrm{MgO}}^{\mathrm{l}}\right)}{\left(\mathrm{a}_{\mathrm{FeO}}^{\mathrm{l}}\right)\left(\mathrm{a}_{\mathrm{MgO}}^{\mathrm{ol}}\right)}=\frac{\left(\mathrm{X}_{\mathrm{FeO}}^{\mathrm{ol}}\right)\left(\mathrm{X}_{\mathrm{MgO}}^{\mathrm{l}}\right)}{\left(\mathrm{X}_{\mathrm{FeO}}^{\mathrm{l}}\right)\left(\mathrm{X}_{\mathrm{MgO}}^{\mathrm{ol}}\right)} \\
& \times \frac{\left(\gamma^{\mathrm{ol}} \mathrm{FeO}_{\mathrm{FeO}}\right)\left(\gamma_{\mathrm{MgO}}^{\mathrm{l}}\right)}{\left(\gamma_{\mathrm{FeO}}^{\mathrm{l}}\right)\left(\gamma^{\mathrm{ol}}{ }_{\mathrm{MgO}}\right)}
\end{aligned}
$$

The ratio of the mole fractions were defined in Roeder and Emslie (1970) as the distribution coefficient. This coefficient was previously studied thermodynamically by Bowen and Schairer (1935). Bradley (1962) revised the equations presented by Bowen and Schairer using different specific heat terms and found that the agreement with Bowen and Schairer's work still was very good.

Several authors (e.g. Roeder and Emslie 1970, Duke 1976) found that this distribution coefficient

$$
\mathrm{K}_{\mathrm{D}}=\frac{\left(\mathrm{X}_{\mathrm{FeO}}^{\mathrm{ol}}\right)\left(\mathrm{X}_{\mathrm{MgO}}{ }_{\mathrm{Hg}}\right)}{\left(\mathrm{X}_{\mathrm{FeO}}^{\mathrm{l}}\right)\left(\mathrm{X}^{\mathrm{ol}}{ }_{\mathrm{MgO}}\right)}
$$

is equal to 0.30 at equilibrium conditions. Other variables such as pressure, oxygen fugacity and temperature have no significant effect. This value is discussed in detail by Roeder and Emslie (1970).

However, the lunar basalt experiments (Longhi et al. 1975, Walker et al. 1976) show a somewhat higher average on $\mathrm{K}_{\mathrm{D}}(0.33)$. 
Table 2. The Fo-content in olivine (mole- $\%$ ) and $\mathrm{K}_{\mathrm{D}}$ values. Columns: 1 . Fo content read directly from $\mathrm{Mg}_{2} \mathrm{SiO}_{4}-\mathrm{Fe}_{2} \mathrm{SiO}_{4}$ diagram. 2. Fo content read from $\mathrm{FeO}^{\mathrm{l}}-\mathrm{MgO}^{\mathrm{l}}$ diagram (Fig. 4). 3. Analyzed olivine composition (Mäkipää 1978). 4. $\mathrm{K}_{\mathrm{D}}=$ $\left(\mathrm{FeO}^{\mathrm{ol}}\right)\left(\mathrm{MgO}^{\mathrm{l}}\right)$

$\left(\mathrm{FeO}^{\mathrm{l}}\right)(\mathrm{MgO})$

ma - macrophenocryst, mi - microphenocryst. Sample location is shown on the map and given in Appendix 1 (Mäkipää 1978).

\begin{tabular}{|c|c|c|c|c|}
\hline Sample No. & 1 & 2 & 3 & 4 \\
\hline 1 & 78.5 & 77.1 & 78.49 & 0.282 \\
\hline 3 & 74.5 & 71.7 & 75.29 & 0.256 \\
\hline $4\left\{\begin{array}{l}\mathrm{ma} \\
\mathrm{mi}\end{array}\right.$ & 82.4 & 82.2 & $\begin{array}{l}83.06 \\
83.14\end{array}$ & $\begin{array}{l}0.284 \\
0.282\end{array}$ \\
\hline $5\left\{\begin{array}{l}\mathrm{ma} \\
\mathrm{mi}\end{array}\right.$ & 79.8 & 78.9 & $\begin{array}{l}82.11 \\
77.62\end{array}$ & $\begin{array}{l}0.250 \\
0.328\end{array}$ \\
\hline $\begin{array}{r}7 \\
10\end{array}$ & $\begin{array}{l}75.2 \\
81.0\end{array}$ & $\begin{array}{l}72.9 \\
80.4\end{array}$ & $\begin{array}{l}75.63 \\
79.04\end{array}$ & $\begin{array}{l}0.266 \\
0.329\end{array}$ \\
\hline $11\left\{\begin{array}{l}\mathrm{ma} \\
\mathrm{mi}\end{array}\right.$ & 85.1 & 84.7 & $\begin{array}{l}88.77 \\
84.99\end{array}$ & $\begin{array}{l}0.211 \\
0.294\end{array}$ \\
\hline $\begin{array}{l}16 \\
17\end{array}$ & $\begin{array}{l}81.0 \\
81.8\end{array}$ & $\begin{array}{l}80.6 \\
81.3\end{array}$ & $\begin{array}{l}80.91 \\
80.49\end{array}$ & $\begin{array}{l}0.297 \\
0.320\end{array}$ \\
\hline $19\left\{\begin{array}{l}\mathrm{ma} \\
\mathrm{mi}\end{array}\right.$ & 82.7 & 82.3 & $\begin{array}{l}83.77 \\
84.01\end{array}$ & $\begin{array}{l}0.271 \\
0.267\end{array}$ \\
\hline $20\left\{\begin{array}{l}\mathrm{ma} \\
\mathrm{mi}\end{array}\right.$ & 83.4 & 83.0 & $\begin{array}{l}84.91 \\
81.17\end{array}$ & $\begin{array}{l}0.260 \\
0.340\end{array}$ \\
\hline $\begin{array}{l}22 \\
24\end{array}$ & $\begin{array}{l}73.4 \\
72.8\end{array}$ & $\begin{array}{l}72.7 \\
71.0\end{array}$ & $\begin{array}{l}69.40 \\
64.99\end{array}$ & $\begin{array}{l}0.361 \\
0.405\end{array}$ \\
\hline $30\left\{\begin{array}{l}\mathrm{ma} \\
\mathrm{mi}\end{array}\right.$ & 84.7 & 84.5 & $\begin{array}{l}85.73 \\
85.58\end{array}$ & $\begin{array}{l}0.271 \\
0.275\end{array}$ \\
\hline $32\left\{\begin{array}{l}\mathrm{ma} \\
\mathrm{mi}\end{array}\right.$ & 85.3 & 85.1 & $\begin{array}{l}86.01 \\
85.91\end{array}$ & $\begin{array}{l}0.277 \\
0.279\end{array}$ \\
\hline $\begin{array}{l}35 \\
38 \\
96\end{array}$ & $\begin{array}{l}84.7 \\
77.9 \\
84.5\end{array}$ & $\begin{array}{l}84.7 \\
77.7 \\
83.2\end{array}$ & $\begin{array}{l}85.23 \\
76.84 \\
85.23\end{array}$ & $\begin{array}{l}0.286 \\
0.320 \\
0.264\end{array}$ \\
\hline $99\left\{\begin{array}{l}\mathrm{ma} \\
\mathrm{mi}\end{array}\right.$ & 80.7 & 79.2 & $\begin{array}{l}80.36 \\
80.94\end{array}$ & $\begin{array}{l}0.275 \\
0.286\end{array}$ \\
\hline $\begin{array}{l}101 \mathrm{~A} \\
101 \mathrm{~B}\end{array}$ & $\begin{array}{l}84.6 \\
78.3\end{array}$ & $\begin{array}{l}83.3 \\
76.6\end{array}$ & $\begin{array}{l}81.06 \\
81.75\end{array}$ & $\begin{array}{l}0.360 \\
0.236\end{array}$ \\
\hline $104\left\{\begin{array}{l}\mathrm{ma} \\
\mathrm{mi}\end{array}\right.$ & 87.4 & 86.5 & $\begin{array}{l}85.44 \\
85.38\end{array}$ & $\begin{array}{l}0.321 \\
0.319\end{array}$ \\
\hline
\end{tabular}

In Roeder and Emslie's (1970) experiments the $\mathrm{K}_{\mathrm{D}}$ values are within 0.06 units $\left(\mathrm{K}_{\mathrm{D}}=\mathbf{0 . 3 0}\right.$, $\mathrm{SD}=0.03$ ) and the $\mathrm{K}_{\mathrm{D}}$ values in the present study (Table 2) between 0.26 and 0.34 are assumed to represent equilibria.

To get better illustration of this equilibria the elemental distribution ( $\mathrm{Fe} / \mathrm{Mg}$ in olivine versus $\mathrm{Fe} / \mathrm{Mg}$ in liquid) is plotted in Fig. 1. The area between the lines presents equilibria in this study and most of the samples fall between those limits.

\section{Olivine geothermometers}

Roeder and Emslie (1970) graphically presented the dependence of olivine composition on the composition of the liquid by plotting the mole per cent $\mathrm{FeO}$ in liquid against the mole per cent $\mathrm{MgO}$ in liquid (Fig. 2). Roeder and Emslie discussed in detail the usefulness and limitations of this diagram. Because the composition of the basalts used here are equal to their experimental materials and there is more than enough $\mathrm{SiO}_{2}$ in liquid to combine with $\mathrm{MgO}$ and $\mathrm{FeO}$ to form olivine, this diagram offers the possibility to estimate the composition of the equilibrium olivine as well as the equilib-

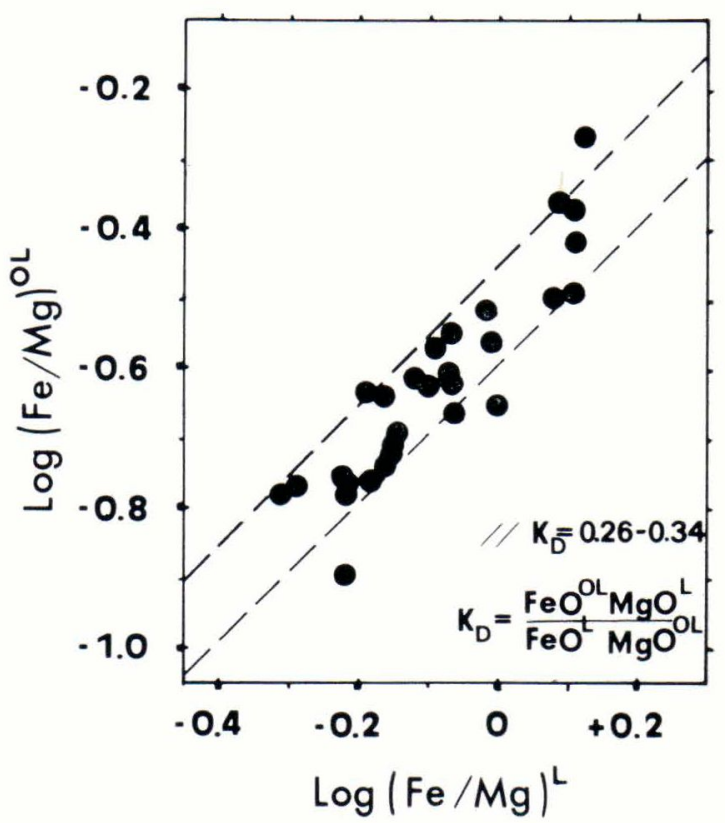

Fig. 1. Log (Fe/Mg) in olivine plotted against $\log (\mathrm{Fe} / \mathrm{Mg})$ in liquid. The area between the lines indicates equilibrium conditions $\left(\mathrm{K}_{\mathrm{D}}=0.26-0.34\right)$ assumed in this study. 
Fig. 2. FeO (mole- $\%)$ in liquid plotted against $\mathrm{MgO}\left(\mathrm{mole}^{-} \%\right.$ ) in liquid to estimate the equilibrium temperatures and the compositions of the equilibrium olivines (Roeder \& Emslie 1970). Numbers refer to the samples (Mäkipää 1978).

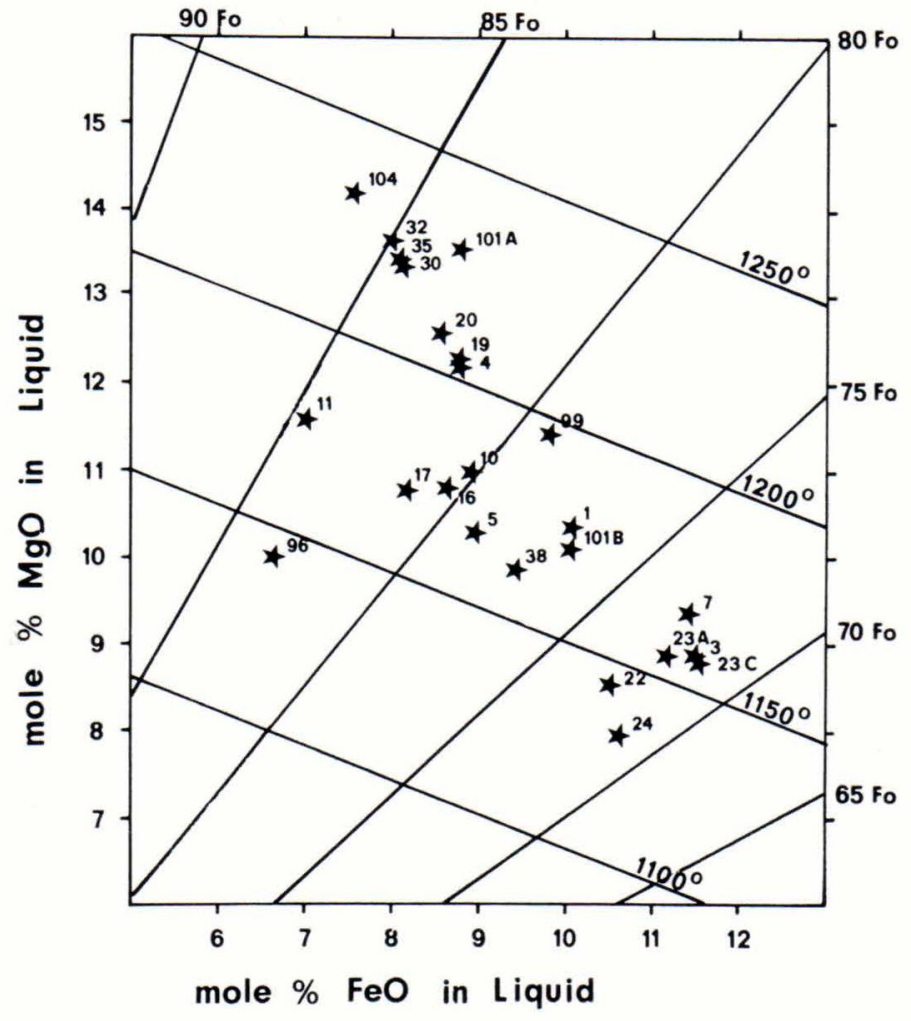

in basic liquids giving the following equations:

$$
\begin{aligned}
& \log \frac{\mathrm{X}_{\mathrm{MgO}}^{\mathrm{ol}}}{\mathrm{X}_{\mathrm{MgO}}^{1}}=\frac{3480}{\mathrm{~T}}-1.70 \\
& \log \frac{\mathrm{X}_{\mathrm{FeO}}^{\mathrm{ol}}}{\mathrm{X}_{\mathrm{FeO}}^{1}}=\frac{3740}{\mathrm{~T}}-2.38 \\
& \log \frac{\mathrm{X}_{\mathrm{MnO}}^{\mathrm{ol}}}{\mathrm{X}_{\mathrm{MnO}}^{1}}=\frac{3850}{\mathrm{~T}}-2.59
\end{aligned}
$$

The equilibrium temperatures calculated using these equations are given in Table 3 . The temperature calculated from the $\mathrm{MgO}$ distribution should be the most accurate. The $\mathrm{FeO}$ temperature is dependent on the $\mathrm{FeO}$

\footnotetext{
content of the magma which can not be measured directly and for $\mathrm{MnO}$ temperature estimates more accurate determinations are needed.

where $\mathrm{X}$ is the mole fraction of the element and $\mathrm{T}$ temperature in $\mathrm{K}^{\circ}$.

Roeder (1974) modified these equations using data on the solubility of ferrous iron
} 
Table 3. Calculated olivine and plagioclase temperatures. OL - olivine (Roeder $1974, \mathrm{Fe} / \mathrm{Mg}$ refer to $\mathrm{FeO}^{1} / \mathrm{MgO}^{1}$ diagram in Roeder and Emslie 1970, Fig. 7). PL - plagioclase (Kudo and Weill 1970, Mathez 1973). ma - macrophenocryst, $\mathrm{mi}$ - microphenocryst. Sample location is shown on the map and given in Appendix 1 (Mäkipää 1978).

\begin{tabular}{|c|c|c|c|c|c|c|c|c|c|c|c|c|c|c|}
\hline \multirow{3}{*}{\multicolumn{2}{|c|}{ Sample No. }} & \multicolumn{13}{|c|}{ calculated temperatures $\left({ }^{\circ} \mathrm{C}\right)$} \\
\hline & & \multicolumn{3}{|c|}{ Roeder 1974} & \multirow{2}{*}{$\begin{array}{l}\text { (OL) } \\
\mathrm{Fe} / \mathrm{Mg}\end{array}$} & \multicolumn{4}{|c|}{$\begin{array}{l}\text { Kudo \& Weill } \\
\left(\mathrm{PL}, \mathrm{P}_{\mathrm{H}_{2} \mathrm{O}}\right)\end{array}$} & \multicolumn{4}{|c|}{ Mathez $\left(\mathrm{PL}, \mathrm{P}_{\mathrm{H}_{2} \mathrm{O}}\right)$} & \multirow{2}{*}{ USED } \\
\hline & & $\mathrm{Mg}$ & $\mathrm{Fe}$ & $\mathrm{Mn}$ & & 0 & $\begin{array}{l}(\mathrm{P} L, \mathrm{~F} \\
0.5\end{array}$ & $\begin{aligned} \mathrm{H}_{2} \mathrm{OI} \\
1.0\end{aligned}$ & 5.0 & 0 & 0.5 & 1.0 & 5.0 & \\
\hline $\mathrm{RE}-1 \quad\{$ & $\left\{\begin{array}{l}\mathrm{mi} \\
\mathrm{ma}\end{array}\right.$ & 1178 & 1206 & 1202 & 1177 & $\begin{array}{l}1212 \\
1264\end{array}$ & $\begin{array}{l}1164 \\
1213\end{array}$ & $\begin{array}{l}1129 \\
1178\end{array}$ & $\begin{array}{l}872 \\
921\end{array}$ & $\begin{array}{l}1177 \\
1223\end{array}$ & $\begin{array}{l}1212 \\
1274\end{array}$ & $\begin{array}{l}1162 \\
1220\end{array}$ & $\begin{array}{l}872 \\
921\end{array}$ & 1190 \\
\hline TH-3 & $\left\{\begin{array}{l}\mathrm{mi} \\
\mathrm{ma}\end{array}\right.$ & 1150 & 1203 & 1182 & 1158 & $\begin{array}{l}1197 \\
1197\end{array}$ & $\begin{array}{l}1150 \\
1150\end{array}$ & $\begin{array}{l}1114 \\
1114\end{array}$ & $\begin{array}{l}851 \\
851\end{array}$ & $\begin{array}{l}1164 \\
1164\end{array}$ & $\begin{array}{l}1194 \\
1194\end{array}$ & $\begin{array}{l}1145 \\
1145\end{array}$ & $\begin{array}{l}851 \\
851\end{array}$ & 1158 \\
\hline $\mathrm{TH}-4 \quad\{$ & $\left\{\begin{array}{l}\mathrm{mi} \\
\mathrm{ma}\end{array}\right.$ & $\begin{array}{l}1200 \\
1204\end{array}$ & $\begin{array}{l}1228 \\
1229\end{array}$ & $\begin{array}{l}1226 \\
1222\end{array}$ & 1203 & $\begin{array}{l}1289 \\
1291\end{array}$ & $\begin{array}{l}1238 \\
1240\end{array}$ & $\begin{array}{l}1204 \\
1206\end{array}$ & $\begin{array}{l}951 \\
953\end{array}$ & $\begin{array}{l}1245 \\
1247\end{array}$ & $\begin{array}{l}1304 \\
1306\end{array}$ & $\begin{array}{l}1249 \\
1251\end{array}$ & $\begin{array}{l}951 \\
953\end{array}$ & 1218 \\
\hline RE-5 & $\left\{\begin{array}{l}\mathrm{mi} \\
\mathrm{ma}\end{array}\right.$ & $\begin{array}{l}1165 \\
1179\end{array}$ & $\begin{array}{l}1223 \\
1170\end{array}$ & $\begin{array}{l}1172 \\
1138\end{array}$ & 1168 & $\begin{array}{l}1209 \\
1262\end{array}$ & $\begin{array}{l}1162 \\
1211\end{array}$ & $\begin{array}{l}1127 \\
1176\end{array}$ & $\begin{array}{l}867 \\
916\end{array}$ & $\begin{array}{l}1175 \\
1221\end{array}$ & $\begin{array}{l}1210 \\
1272\end{array}$ & $\begin{array}{l}1160 \\
1218\end{array}$ & $\begin{array}{l}867 \\
916\end{array}$ & 1168 \\
\hline TH-7 & $\mathrm{mi}$ & 1160 & 1203 & 1169 & 1168 & 1215 & 1167 & 1132 & 870 & 1180 & 1216 & 1166 & 870 & 1166 \\
\hline $\mathrm{TH}-10 \quad\{$ & $\left\{\begin{array}{l}\mathrm{mi} \\
\mathrm{ma}\end{array}\right.$ & 1194 & 1182 & 1149 & 1184 & $\begin{array}{l}1210 \\
1273\end{array}$ & $\begin{array}{l}1163 \\
1222\end{array}$ & $\begin{array}{l}1129 \\
1188\end{array}$ & $\begin{array}{l}874 \\
932\end{array}$ & $\begin{array}{l}1176 \\
1231\end{array}$ & $\begin{array}{l}1210 \\
1285\end{array}$ & $\begin{array}{l}1161 \\
1231\end{array}$ & $\begin{array}{l}874 \\
932\end{array}$ & 1188 \\
\hline $\mathrm{RE}-11$ & $\left\{\begin{array}{l}\mathrm{mi} \\
\mathrm{ma}\end{array}\right.$ & $\begin{array}{l}1182 \\
1171\end{array}$ & $\begin{array}{l}1199 \\
1276\end{array}$ & $\begin{array}{l}1308 \\
1310\end{array}$ & 1180 & $\begin{array}{l}1246 \\
1247\end{array}$ & $\begin{array}{l}1198 \\
1199\end{array}$ & $\begin{array}{l}1164 \\
1165\end{array}$ & $\begin{array}{l}915 \\
916\end{array}$ & $\begin{array}{l}1208 \\
1209\end{array}$ & $\begin{array}{l}1253 \\
1254\end{array}$ & $\begin{array}{l}1202 \\
1203\end{array}$ & $\begin{array}{l}915 \\
916\end{array}$ & 1190 \\
\hline $\mathrm{RE}-16 \quad$ & $\left\{\begin{array}{l}\mathrm{mi} \\
\mathrm{ma}\end{array}\right.$ & 1181 & 1196 & 1211 & 1177 & $\begin{array}{l}1221 \\
1260\end{array}$ & $\begin{array}{l}1174 \\
1210\end{array}$ & $\begin{array}{l}1139 \\
1176\end{array}$ & $\begin{array}{l}889 \\
916\end{array}$ & $\begin{array}{l}1186 \\
1220\end{array}$ & $\begin{array}{l}1224 \\
1270\end{array}$ & $\begin{array}{l}1174 \\
1217\end{array}$ & $\begin{array}{l}884 \\
919\end{array}$ & 1196 \\
\hline $\mathrm{RE}-17$ \{ & $\left\{\begin{array}{l}\mathrm{mi} \\
\mathrm{ma}\end{array}\right.$ & 1176 & 1173 & 1191 & 1173 & $\begin{array}{l}1208 \\
1204\end{array}$ & & $\begin{array}{l}1126 \\
1121\end{array}$ & & & $\begin{array}{l}1208 \\
1203\end{array}$ & $\begin{array}{l}1158 \\
1153\end{array}$ & 65 & 1180 \\
\hline $\mathrm{RE}-19$ & $\left\{\begin{array}{l}\mathrm{mi} \\
\mathrm{ma}\end{array}\right.$ & & $\begin{array}{l}1246 \\
1242\end{array}$ & $\begin{array}{l}1189 \\
1269\end{array}$ & 1 & $\begin{array}{l}1239 \\
1234\end{array}$ & & & & & & $\begin{array}{l}1194 \\
1189\end{array}$ & & 1210 \\
\hline $\mathrm{RE}-20$ & $\left\{\begin{array}{l}\mathrm{mi} \\
\mathrm{ma}\end{array}\right.$ & $\begin{array}{l}1215 \\
1202\end{array}$ & $\begin{array}{l}1193 \\
1250\end{array}$ & $\begin{array}{l}1158 \\
1186\end{array}$ & 1209 & 1244 & 1196 & 1163 & 916 & 1207 & 1250 & 1200 & 916 & 206 \\
\hline GR-22 & $\left\{\begin{array}{c}\mathrm{mi} \\
\mathrm{ma}\end{array}\right.$ & 1163 & 1133 & 1155 & 1144 & $\begin{array}{l}1203 \\
1242\end{array}$ & $\begin{array}{l}1155 \\
1191\end{array}$ & $\begin{array}{l}1118 \\
1155\end{array}$ & $\begin{array}{l}849 \\
886\end{array}$ & $\begin{array}{l}1169 \\
1203\end{array}$ & $\begin{array}{l}1202 \\
1248\end{array}$ & $\begin{array}{l}1151 \\
1194\end{array}$ & $\begin{array}{l}849 \\
886\end{array}$ & 1154 \\
\hline GR-23A & $\mathrm{mi}$ & 1161 & 1167 & 1091 & 1155 & 1212 & 1163 & 1127 & 858 & 1177 & 1213 & 1161 & 858 & 1161 \\
\hline GR-23C & $\left\{\begin{array}{c}\mathrm{mi} \\
\mathrm{ma}\end{array}\right.$ & 1168 & 1157 & 1168 & 1157 & $\begin{array}{l}1197 \\
1189\end{array}$ & $\begin{array}{l}1150 \\
1142\end{array}$ & $\begin{array}{l}1115 \\
1107\end{array}$ & $\begin{array}{l}851 \\
842\end{array}$ & $\begin{array}{l}1165 \\
1158\end{array}$ & $\begin{array}{l}1196 \\
1185\end{array}$ & $\begin{array}{l}1145 \\
1136\end{array}$ & $\begin{array}{l}851 \\
842\end{array}$ & 1163 \\
\hline $\mathrm{KE}-24$ & $\left\{\begin{array}{l}\mathrm{mi} \\
\mathrm{ma}\end{array}\right.$ & 1165 & 1108 & 1098 & 1130 & $\begin{array}{l}1221 \\
1206\end{array}$ & $\begin{array}{l}1172 \\
1158\end{array}$ & $\begin{array}{l}1136 \\
1122\end{array}$ & $\begin{array}{l}871 \\
856\end{array}$ & $\begin{array}{l}1185 \\
1172\end{array}$ & $\begin{array}{l}1224 \\
1205\end{array}$ & $\begin{array}{l}1171 \\
1154\end{array}$ & $\begin{array}{l}871 \\
856\end{array}$ & 1130 \\
\hline $\mathrm{HE}-30$ & $\left\{\begin{array}{l}\mathrm{mi} \\
\mathrm{ma}\end{array}\right.$ & $\begin{array}{l}1221 \\
1220\end{array}$ & $\begin{array}{l}1255 \\
1257\end{array}$ & $\begin{array}{l}1245 \\
1220\end{array}$ & 1220 & $\begin{array}{l}1249 \\
1238\end{array}$ & $\begin{array}{l}1201 \\
1190\end{array}$ & $\begin{array}{l}1167 \\
1157\end{array}$ & $\begin{array}{l}920 \\
910\end{array}$ & $\begin{array}{l}1211 \\
1201\end{array}$ & $\begin{array}{l}1256 \\
1243\end{array}$ & $\begin{array}{l}1205 \\
1193\end{array}$ & $\begin{array}{l}920 \\
910\end{array}$ & 1235 \\
\hline $\mathrm{HE}-32$ & $\left\{\begin{array}{c}\mathrm{mi} \\
\mathrm{ma}\end{array}\right.$ & $\begin{array}{l}1228 \\
1226\end{array}$ & $\begin{array}{l}1257 \\
1257\end{array}$ & $\begin{array}{l}1240 \\
1242\end{array}$ & 1225 & 1256 & 1208 & 1175 & 931 & 1217 & 1264 & 1214 & 931 & 1238 \\
\hline HE-35 & $\mathrm{mi}$ & 1223 & 1246 & 1250 & 1222 & 1256 & 1208 & 1175 & 931 & 217 & 1264 & 1214 & 931 & 1236 \\
\hline LA-38 & $\mathrm{mi}$ & 1171 & 1169 & 1133 & 1163 & 1217 & 1169 & 1134 & 873 & 1182 & 1218 & 1169 & 873 & 1168 \\
\hline TJ-96 & $\left\{\begin{array}{l}\mathrm{mi} \\
\mathrm{ma}\end{array}\right.$ & 1158 & 1203 & 1166 & 1144 & $\begin{array}{l}1213 \\
1165\end{array}$ & $\begin{array}{l}1166 \\
1121\end{array}$ & $\begin{array}{l}1131 \\
1086\end{array}$ & $\begin{array}{l}873 \\
827\end{array}$ & $\begin{array}{l}1179 \\
1137\end{array}$ & $\begin{array}{l}1214 \\
1157\end{array}$ & $\begin{array}{l}1164 \\
1110\end{array}$ & $\begin{array}{l}873 \\
827\end{array}$ & 1156 \\
\hline TJ-99 & $\left\{\begin{array}{l}\mathrm{mi} \\
\mathrm{ma}\end{array}\right.$ & $\begin{array}{l}1201 \\
1198\end{array}$ & $\begin{array}{l}1225 \\
1154\end{array}$ & $\begin{array}{l}1213 \\
1200\end{array}$ & 1195 & $\begin{array}{l}1253 \\
1156\end{array}$ & $\begin{array}{l}1205 \\
1114\end{array}$ & $\begin{array}{l}1171 \\
1080\end{array}$ & $\begin{array}{l}922 \\
833\end{array}$ & $\begin{array}{l}1214 \\
1131\end{array}$ & $\begin{array}{l}1261 \\
1147\end{array}$ & $\begin{array}{l}1210 \\
1103\end{array}$ & $\begin{array}{l}922 \\
833\end{array}$ & 1207 \\
\hline TJ-101B & $\mathrm{mi}$ & 1160 & 1248 & 1302 & 1177 & 1200 & 1156 & 1123 & 884 & 1168 & 1198 & 1152 & 884 & 1169 \\
\hline TJ-101A & $\mathrm{mi}$ & 1242 & 1203 & 1282 & 1228 & 1256 & 1207 & 1172 & 921 & 1216 & 1264 & 1212 & 921 & 1224 \\
\hline TJ-104 & $\left\{\begin{array}{c}\mathrm{mi} \\
\mathrm{ma}\end{array}\right.$ & 1238 & 1228 & 1230 & 1230 & $\begin{array}{l}1301 \\
1289\end{array}$ & $\begin{array}{l}1250 \\
1239\end{array}$ & $\begin{array}{l}1217 \\
1206\end{array}$ & $\begin{array}{l}973 \\
963\end{array}$ & $\begin{array}{l}1257 \\
1247\end{array}$ & $\begin{array}{l}1317 \\
1303\end{array}$ & $\begin{array}{l}1263 \\
1252\end{array}$ & $\begin{array}{l}973 \\
963\end{array}$ & 1231 \\
\hline
\end{tabular}


Leeman and Scheidegger (1977) also studied the use of the olivine-liquid distribution as a geothermometer. They concluded that the apparent olivine distribution coefficients found for their samples were reasonable approximations to the true equilibrium values. They also emphasized that factors such as liquid temperature and composition might have significant influence on the partition coefficients in nature.

However, values derived using their geothermometer equation for $\mathrm{Mg}$

$$
\ln \mathrm{D}_{\mathrm{Mg}}=\frac{8916}{\mathrm{~T}}-4.29
$$

can be compared with the temperatures derived using Roeder and Emslie's (1970) equation.

As seen from the Fig. 3 all temperatures calculated using Leeman and Scheidegger's geothermometer are systematically about $5{ }^{\circ} \mathrm{C}$ higher than those calculated from Roeder and Emslie. The disequilibrium samples stand out very clear.

\section{Plagioclase-liquid combination}

\section{General}

In his classic investigation of melting in the plagioclase system Bowen (1913) demonstrated that albite $\left(\mathrm{NaAlSi}_{3} \mathrm{O}_{8}\right)$ and anorthite $\left(\mathrm{CaAl}_{2} \mathrm{Si}_{2} \mathrm{O}_{8}\right)$ form a continuous solid solution series at high temperatures. The experimental relationship found between the compositions of liquid and coexisting solid solution and temperature was satisfactorily accounted for by Bowen by assuming that both the liquid and solid solutions were ideal, i.e., that the activities of the $\mathrm{Ab}$ and $\mathrm{An}$ components were equal to their mole fraction.

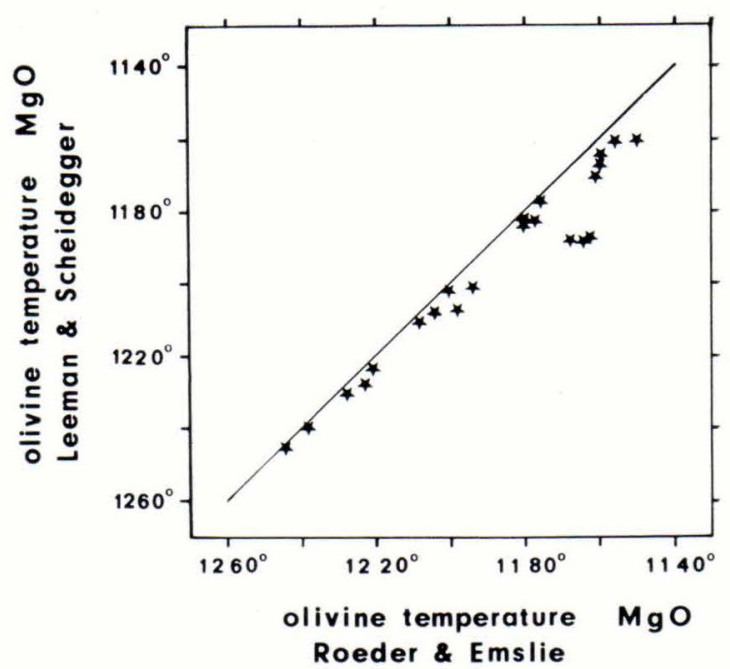

Fig. 3. Calculated olivine MgO-temperatures (after Roeder and Emslie 1970) plotted against calculated olivine Mg-temperatures (after Leeman and Scheidegger 1977).

Some attempts have been made (e.g. Larsen and Irving 1938, Carmichael 1960) to compare Bowen's results in the system directly to magmatic systems. The activities of An and $\mathrm{Ab}$ are replaced with their normative concentrations calculated from the liquid compositions. These attempts have not been very successful; the reasons for which are discussed by Botting et al. (1966).

Bowen's (1915) work on the 'haplobasaltichaplodioritic' system provides a simple model to determine the composition of equilibrium plagioclase crystals. Lack of knowledge of variables such as total pressure and fucacities of important volatile components such as water and oxygen has limited the use of these results. However, several authors (e.g. Kudo and Weill 1970) pointed out the suitability of this haplobasaltic system to estimate approximate equilibria. Therefore the compositions in the present study were also read directly from the phase diagrams An$\mathrm{Ab}-\mathrm{Di}$ and An-Ab. The purpose of this is 
Table 4. The An-content in plagioclase (mole-\%). Columns: 1 . An content read directly from Ab-An$\mathrm{Di}$ and Ab-An diagrams. 2. Analyzed plagioclase composition (Mäkipää 1978). ma - macrophenocryst, mi - microphenocryst. Sample location is shown on the map and given in Appendix 1 (Mäkipää 1978).

\begin{tabular}{|c|c|c|}
\hline Sample No. & 1 & 2 \\
\hline $1\left\{\begin{array}{l}\mathrm{ma} \\
\mathrm{mi}\end{array}\right.$ & 84.8 & $\begin{array}{l}90.07 \\
81.64\end{array}$ \\
\hline $3\left\{\begin{array}{l}\mathrm{ma} \\
\mathrm{mi}\end{array}\right.$ & 85.2 & $\begin{array}{l}76.96 \\
75.75\end{array}$ \\
\hline $4\left\{\begin{array}{c}\mathrm{ma} \\
\mathrm{mi}\end{array}\right.$ & 87.2 & $\begin{array}{l}92.28 \\
92.14\end{array}$ \\
\hline $5\left\{\begin{array}{l}\mathrm{ma} \\
\mathrm{mi}\end{array}\right.$ & 82.8 & $\begin{array}{l}89.58 \\
80.38\end{array}$ \\
\hline 7 & 83.5 & 76.64 \\
\hline $10\left\{\begin{array}{l}\mathrm{ma} \\
\mathrm{mi}\end{array}\right.$ & 85.4 & $\begin{array}{l}90.67 \\
80.15\end{array}$ \\
\hline $11\left\{\begin{array}{l}\mathrm{ma} \\
\mathrm{mi}\end{array}\right.$ & 86.0 & $\begin{array}{l}86.39 \\
86.32\end{array}$ \\
\hline $16\left\{\begin{array}{l}\mathrm{ma} \\
\mathrm{mi}\end{array}\right.$ & 84.9 & $\begin{array}{l}89.71 \\
83.71\end{array}$ \\
\hline $17\left\{\begin{array}{l}\mathrm{ma} \\
\mathrm{mi}\end{array}\right.$ & 83.3 & $\begin{array}{l}80.70 \\
82.61\end{array}$ \\
\hline $19\left\{\begin{array}{l}\mathrm{ma} \\
\mathrm{mi}\end{array}\right.$ & 86.6 & $\begin{array}{l}82.66 \\
84.63\end{array}$ \\
\hline 20 & 86.3 & 83.00 \\
\hline $22\left\{\begin{array}{l}\mathrm{ma} \\
\mathrm{mi}\end{array}\right.$ & 80.4 & $\begin{array}{l}88.07 \\
78.30\end{array}$ \\
\hline $24\left\{\begin{array}{l}\mathrm{ma} \\
\mathrm{mi}\end{array}\right.$ & 80.0 & $\begin{array}{l}66.77 \\
71.37\end{array}$ \\
\hline $30\left\{\begin{array}{l}\mathrm{ma} \\
\mathrm{mi}\end{array}\right.$ & 88.0 & $\begin{array}{l}86.01 \\
87.74\end{array}$ \\
\hline 32 & 88.3 & 87.34 \\
\hline 35 & 88.6 & 87.85 \\
\hline 38 & 82.3 & 77.30 \\
\hline $96\left\{\begin{array}{l}\mathrm{ma} \\
\mathrm{mi}\end{array}\right.$ & 86.5 & $\begin{array}{l}59.81 \\
83.52\end{array}$ \\
\hline $99\left\{\begin{array}{l}\mathrm{ma} \\
\mathrm{mi}\end{array}\right.$ & 86.1 & $\begin{array}{l}50.99 \\
86.05\end{array}$ \\
\hline $101 \mathrm{~A}$ & 91.0 & 82.92 \\
\hline $101 \mathrm{~B}$ & 85.0 & 85.51 \\
\hline $104\left\{\begin{array}{l}\mathrm{ma} \\
\mathrm{mi}\end{array}\right.$ & 89.1 & $\begin{array}{l}91.11 \\
92.10\end{array}$ \\
\hline
\end{tabular}

only to get some idea how far from or close to the equilibria the analyzed plagioclase crystals are.
The measured values (Table 4) compared with the analyzed ones clearly show that all the microphenocrysts are in approximate equilibrium. Almost all the macrophenocrysts appear to be far from the equilibria.

Formulation of a plagioclase geothermometer

The first successful attempt at formulating a plagioclase geothermometer is that of Kudo and Weill (1970), who considered the exchange reaction:

$$
\begin{aligned}
& \left(\mathrm{NaSiO}_{2.5}\right) \mathrm{AlSi}_{2} \mathrm{O}_{5.5}+\left(\mathrm{CaAlO}_{2.5}\right) \mathrm{AlSiO}_{2} \mathrm{O}_{5.5} \\
& \text { plagioclase liquid } \\
& =\left(\mathrm{NaSiO}_{2.5}\right) \mathrm{AlSi}_{2} \mathrm{O}_{5.5}+\left(\mathrm{CaAlO}_{2.5}\right) \mathrm{AlSi}_{2} \mathrm{O}_{5.5} \\
& \text { plagioclase liquid }
\end{aligned}
$$

The resultant equilibrium condition, assuming the solid solution to be ideal, is

$$
-\frac{\mu^{\circ}}{\mathrm{RT}}=\ln \lambda / \delta+\ln \gamma_{\mathrm{Na}} \gamma_{\mathrm{Si}} / \gamma_{\mathrm{Ca}} \gamma_{\mathrm{Al}}
$$

where $\mu^{\circ}=\mu_{\mathrm{An}}^{\circ}-\mu_{\mathrm{Ab}}^{\circ}+\mu_{\mathrm{Na}}^{\circ}+\mu_{\mathrm{Si}}^{\circ}-\mu_{\mathrm{Ca}}^{\circ}$ $-\mu_{\mathrm{Al}}{ }^{\circ} \mu^{\circ}=$ chemical potential of the specific element, $\mathrm{T}$ is temperature in Kelvin, $\delta=$ $\mathrm{X}_{\mathrm{Ab}} / \mathrm{X}_{\mathrm{An}}, \lambda=\mathrm{X}_{\mathrm{Na}} \mathrm{X}_{\mathrm{Si}} / \mathrm{X}_{\mathrm{Ca}} \mathrm{X}_{\mathrm{Al}}$ and $\gamma=$ activity coefficient, and suscripts denote the atomic fraction (X) of an element in the melt or mole fractions of $\mathrm{Ab}$ and $\mathrm{An}$ in plagioclase.

Because the values of the activity coefficients were unknown, Kudo and Weill (1970) assumed that the activity coefficients take a form similar to that found for regular solutions:

$$
\ln \gamma=\mathrm{C} \varnothing / \mathrm{T},
$$

where $\mathrm{C}$ is a constant empirically evaluated at $1.29 \times 10^{4} \mathrm{~T}^{-1}$ and $\varnothing$ is the atomic fraction of the elements in the melt.

Kudo and Weill (1970) derived the following set of linear regression curves for different conditions of crystallization: 
$\ln \lambda / \delta+1.29 \times 10^{4} \varnothing / \mathrm{T}=10.34 \times 10^{-3} \mathrm{~T}-17.24($ dry $)$

$\ln \lambda / \delta+1.29 \times 10^{4} \varnothing / \mathrm{T}=11.05 \times 10^{-3} \mathrm{~T}-17.86\left(\mathrm{P}_{2} \mathrm{O}=0.5 \mathrm{~kb}\right)$

$\ln \lambda / \delta+1.29 \times 10^{4} \varnothing / \mathrm{T}=11.14 \times 10^{-3} \mathrm{~T}-17.67\left(\mathrm{P}_{\mathrm{H}_{2} \mathrm{O}}=1.0 \mathrm{~kb}\right)$

$\ln \lambda / \delta+1.29 \times 10^{4} \varnothing / \mathrm{T}=12.18 \times 10^{-3} \mathrm{~T}-16.63\left(\mathrm{P}_{\mathrm{H}_{2} \mathrm{O}}=5.0 \mathrm{~kb}\right)$

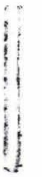

Mathez (1973) modified the Kudo and Weill geothermometer by using $\mathrm{Ab}$ and An activity ratios in the calculations. Mathez pointed out that the Kudo and Weill 'dry' equation gives generally higher temperatures than the experimental values. This divergence may result because the 'dry' equation is generated from plagioclase-liquid equilibrium data for granites in the system An-Ab. Therefore Mathez calculated a new 'dry' equation using available basalt data:

$\ln \lambda / \delta+1.29 \times 10^{4} \varnothing / \mathrm{T}=11.05 \times 10^{-3} \mathrm{~T}-17.98$

The calculated temperatures according to the above equation are in closer agreement with the experimental data than those calculated from Kudo and Weill's 'dry' equation.

By substituting $\left(\mathrm{X}_{\mathrm{Ab} \gamma_{\mathrm{Ab}}}\right) /\left(\mathrm{X}_{\mathrm{An}} \gamma_{\mathrm{An}}\right)$ for $\delta$ in the equation $\gamma_{\mathrm{Ab}} / \gamma_{\mathrm{An}}$ can be calculated from the experimental results. Fig. 4 compares values of $\gamma_{\mathrm{Ab}} / \gamma_{\mathrm{An}}$ calculated using this equation for the experimental data with similar values calculated from the data of Orville (1972).

This Mathez's modification gives the following 'new' equations for different conditions of crystallization:

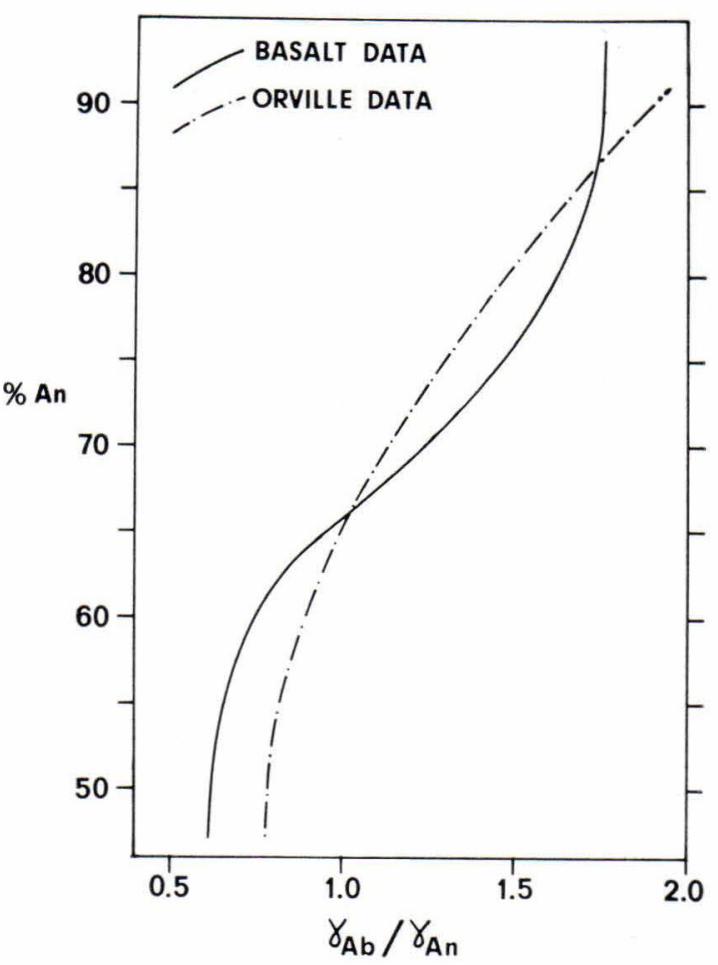

Fig. 4. Comparison of values of $\gamma_{\mathrm{Ab}} / \gamma_{\mathrm{An}}$ as a function of plagioclase composition (Mathez 1973, Fig. 3).

$$
\begin{aligned}
& \ln \lambda / \delta+1.29 \times 10^{4} \varnothing / \mathrm{T}=11.76 \times 10^{-3} \mathrm{~T}-19.01(\mathrm{dry}) \\
& \ln \lambda / \delta+1.29 \times 10^{4} \varnothing / \mathrm{T}=8.97 \times 10^{-3} \mathrm{~T}-15.21\left(\mathrm{P}_{2} \mathrm{O}=0.5 \mathrm{~kb}\right) \\
& \ln \lambda / \delta+1.29 \times 10^{4} \varnothing / \mathrm{T}=9.60 \times 10^{-3} \mathrm{~T}-15.76\left(\mathrm{P}_{\mathrm{H}_{2} \mathrm{O}}=1.0 \mathrm{~kb}\right) \\
& \ln \lambda / \delta+1.29 \times 10^{4} \varnothing / \mathrm{T}=12.18 \times 10^{-3} \mathrm{~T}-16.63\left(\mathrm{P}_{\mathrm{H}_{2} \mathrm{O}}=5.0 \mathrm{~kb}\right)
\end{aligned}
$$

The required input data are $\lambda=\mathrm{x}_{\mathrm{Na}} \mathrm{X}_{\mathrm{Si}} /$ $\mathrm{X}_{\mathrm{Ca}} \mathrm{X}_{\mathrm{Al}}$ and $\varnothing=\mathrm{X}_{\mathrm{Ca}}+\mathrm{X}_{\mathrm{Al}}-\mathrm{X}_{\mathrm{Si}}-\mathrm{X}_{\mathrm{Na}}$, where $\mathrm{X}=$ atomic fraction of the element in the melt and $\delta=\mathrm{X}_{\mathrm{Ab}} \gamma_{\mathrm{Ab}} / \mathrm{X}_{\mathrm{An}} \gamma_{\mathrm{An}}$, where $\mathrm{X}_{\mathrm{Ab}} / \mathrm{X}_{\mathrm{An}}$ is the plagioclase composition and $\gamma_{\mathrm{Ab}} / \gamma_{\mathrm{An}}$ the corresponding activity coefficients.

\section{Equilibrium distribution coefficient}

The elemental partition coefficient. Drake (1975) suggested the use of the major element distribution versus temperature to estimate equilibria. Elemental partition coefficients 
$\mathrm{D}_{\mathrm{Ca}}, \mathrm{D}_{\mathrm{Na}}, \mathrm{D}_{\mathrm{Al}}$ and $\mathrm{D}_{\mathrm{Si}}$ where $\mathrm{D}_{\mathrm{Ca}}=$ (weight of $\mathrm{Ca}$ in plagioclase)/(weight of $\mathrm{Ca}$ in liquid), etc. are given in Table 5 together with equi-

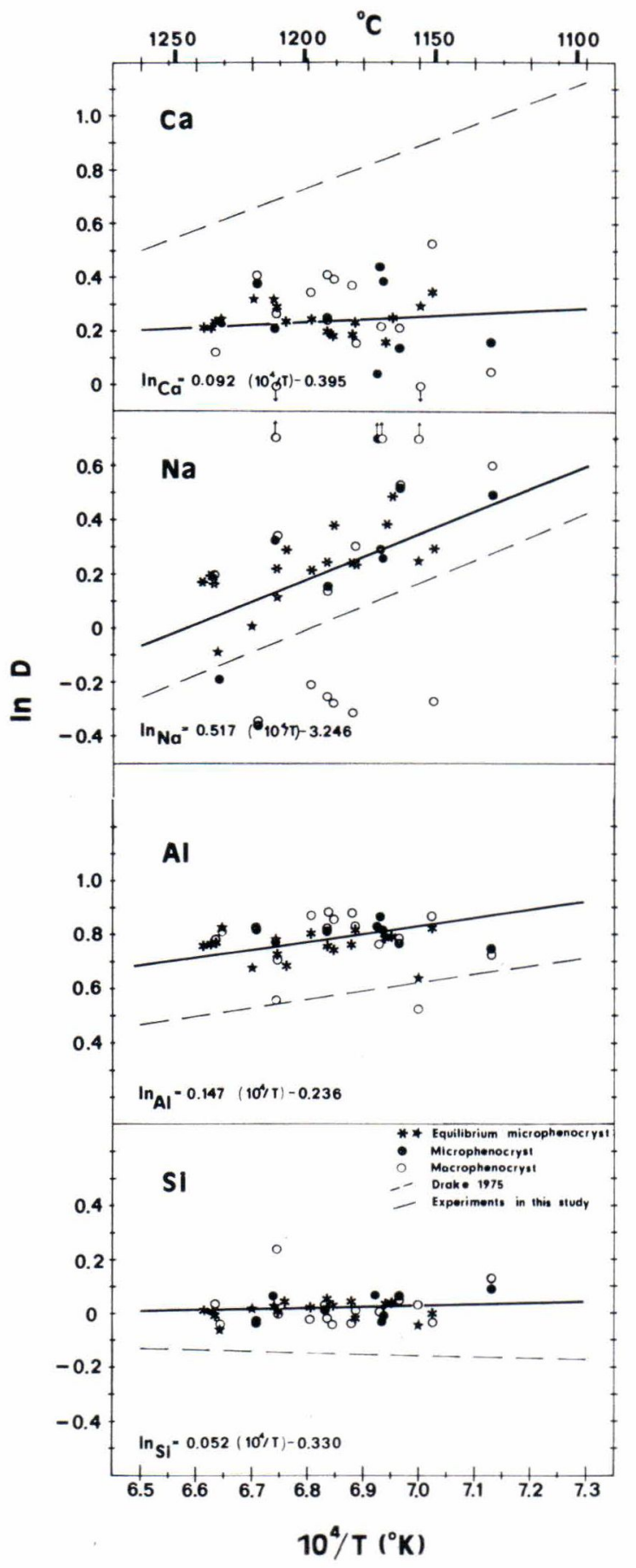

librium constants $(\mathrm{K})$ for the reactions discussed later. The different partition coefficients are plotted in Fig. 5 against the calculated olivine temperature. The difference between the micro- and macrophenocrysts is very apparent. All the regression lines in Drake's experiments are very different from those found here.

Drake (1975) in his discussion made the following remarks: $\mathrm{D}_{\mathrm{Ca}}$ is greater than unity and is strongly dependent upon temperature, $\mathrm{D}_{\mathrm{Na}}$ is less than unity above $1200{ }^{\circ} \mathrm{C}$ and is strongly dependent upon temperature, $\mathrm{D}_{\mathrm{Al}}$ is always greater than unity and is relatively insensitive to temperature and $\mathrm{D}_{\mathrm{Si}}$ is always less than unity and is invariant with temperature.

The present experimental data shows very strong positive correlation between $\mathrm{D}_{\mathrm{Na}}$ and temperature. The difference between Drake's (1975) experiments and the present data is caused of different bulk compositions, which in Drake's experiments were unlike those used in the present study. The regression lines derived from the present experimental data correlate well with the hyaloclastites studied.

The equilibrium constants. The distribution of major elements in plagioclase have been calculated in terms of six reactions (defined as K-values in Drake 1976):

$$
\begin{aligned}
& \mathrm{K}_{5}=\mathrm{CaO}(1)+2 \mathrm{AlO}_{1.5}(1)+2 \mathrm{SiO}_{2}(1) \\
& \rightleftharpoons \mathrm{CaAl}_{2} \mathrm{Si}_{2} \mathrm{O}_{8}(\mathrm{p}) \\
& \mathrm{K}_{6}=\mathrm{NaO}_{0.5}(1)+\mathrm{AlO}_{1.5}(1)+3 \mathrm{SiO}_{2} \\
& \rightleftharpoons \mathrm{NaAlSi}_{3} \mathrm{O}_{8}(\mathrm{p})
\end{aligned}
$$

Fig. 5. Regression curves for In $\mathrm{D}_{\mathrm{Ca}}$, In $\mathrm{D}_{\mathrm{Na}}$, In $\mathrm{D}_{\mathrm{Al}}$, ln $\mathrm{D}_{\mathrm{Si}}$ versus $1 / \mathrm{T}\left({ }^{\circ} \mathrm{K}\right) . \mathrm{D}_{\mathrm{Ca}}=$ (weight of $\mathrm{Ca}$ in plagioclase)/(weight of $\mathrm{Ca}$ in liquid), etc. $\star *=$ plagioclase assumed equilibrium $\bigcirc=$ plagioclase macrophenocryst $\bullet$ = plagioclase microphenocryst. Dashed lines indicate equilibria in Drake's (1975) experiments. Equations and solid lines are fitted by method of least squares to present experimental data only. 
$\mathrm{K}_{7}=\mathrm{CaAl}_{2} \mathrm{O}_{4}(1)+2 \mathrm{SiO}_{2}(1)$

$\rightleftharpoons \mathrm{CaAl}_{2} \mathrm{Si}_{2} \mathrm{O}_{8}(\mathrm{p})$

$\mathrm{K}_{8}=\mathrm{NaAlO}_{2}(1)+3 \mathrm{SiO}_{2}(1)$

$\rightleftharpoons \mathrm{NaAlSi}_{3} \mathrm{O}_{8}(\mathrm{p})$

$\mathrm{K}_{9}=\mathrm{CaO}(1)+\mathrm{AlO}_{1.5}(1)+\mathrm{NaAlSi}_{3} \mathrm{O}_{8}(\mathrm{p})$

$\rightleftharpoons \mathrm{NaO}_{0.5}(1)$

$+\mathrm{SiO}_{2}(1)+\mathrm{CaAl}_{2} \mathrm{Si}_{2} \mathrm{O}_{8}(\mathrm{p})$

$\mathrm{K}_{10}=\mathrm{CaAl}_{2} \mathrm{O}_{4}(1)+\mathrm{NaAlSi}_{3} 0_{8}(\mathrm{p})$

$\rightleftharpoons \mathrm{NaAlO}_{2}(1)+\mathrm{SiO}_{2}(1)$

$+\mathrm{CaAl}_{2} \mathrm{Si}_{2} \mathrm{O}_{8}(\mathrm{p})$,

where (1) refers to liquid and $(p)$ to plagioclase.

The equilibrium constant for reaction $\left(\mathrm{K}_{8}\right)$ involving the equilibrium of albite with the melt component $\mathrm{NaAlO}_{2}$ correlates strongly with reciprocal temperature. However the K-values (Table 5) show similar difference compared with Drake's experiments as the partition coefficients do (Figs. 6 and 7). However the equilibrium plagioclases distinguish the disequilibrium ones.

\section{Clinopyroxene-liquid combination}

Several authors (e.g. Mysen and Boettcher 1975) pointed out that geochemical parameters such as $\mathrm{Fe} / \mathrm{Mg}$ in pyroxene are not suitable as indicators of temperature and pressure because the value increases isothermally with decreasing pressure and is dependent on the liquid composition. Because the compositions of basalts studied can be taken to be constant the distribution of iron

Fig. 6. Effect of pressure on equilibrium constants $\mathrm{K}_{5}, \mathrm{~K}_{6}$ and $\mathrm{K}_{9}$ (see text). Symbols are the same as in Fig. 5. Dashed lines: $\mathrm{D}=$ equilibrium line at 1 atm drawn from Drake (1975), $2 \mathrm{~kb}=$ calculated from experiments of Piwinskii (1968) at $\mathrm{P}_{\mathrm{H} 2 \mathrm{O}}=2 \mathrm{~kb}, 5 \mathrm{~kb}=$ calculated from synthetic system at $\mathrm{P}_{\mathrm{H}_{2} \mathrm{O}}=5 \mathrm{~kb}$ (Yoder et al. 1957). Solid lines are the best fittings by method of least squares to hyaloclastite data only. and magnesium between olivine and clinopyroxene (Duke 1976) might provide a sensitive test for clinopyroxene equilibrium (Fig. 8).

Since $\mathrm{K}^{\mathrm{ol} / \mathrm{liq}} \mathrm{D}(\mathrm{Fe} / \mathrm{Mg})$ is independent on temperature and $\mathrm{Fe} / \mathrm{Mg}$ in clinopyroxene depends on pressure and liquid composition, the

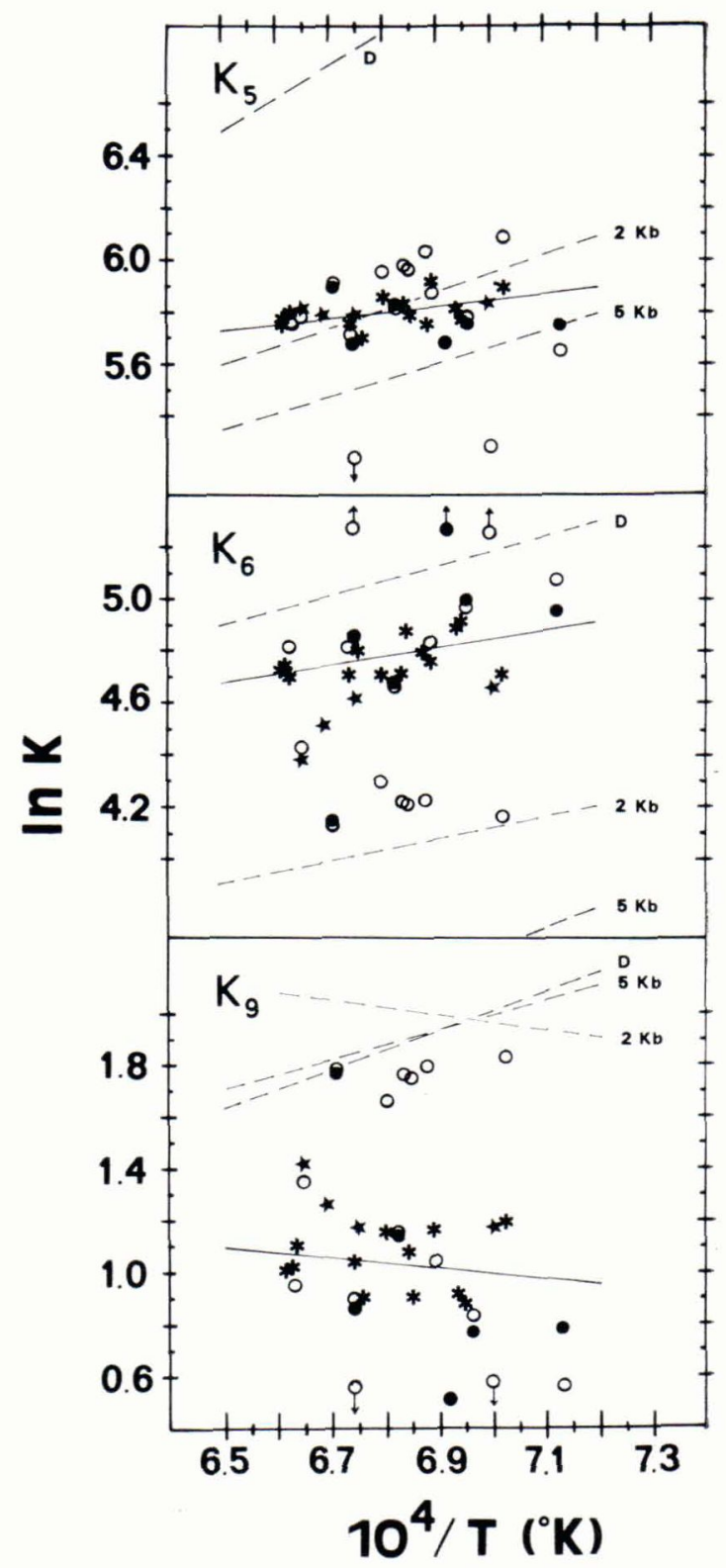


Table 5. The elemental partition coefficients and equilibrium constants for the plagioclase-liquid pair. $\mathrm{D}_{\mathrm{Ca}}$, etc. and $\mathrm{K}_{5}$, etc. see text. $\mathrm{ma}-$ macrophenocryst, $\mathrm{mi}-$ microphenocryst. Sample location is shown on the map and given in Appendix 1 (Mäki pää 1978).

\begin{tabular}{|c|c|c|c|c|c|c|c|c|c|c|c|}
\hline \multirow{2}{*}{\multicolumn{2}{|c|}{ Sample No. }} & \multicolumn{10}{|c|}{ natural logarithm } \\
\hline & & $\mathrm{D}_{\mathrm{Ca}}$ & $\mathrm{D}_{\mathrm{Na}}$ & $\mathrm{D}_{\mathrm{Al}}$ & $\mathrm{D}_{\mathrm{Si}}$ & $\mathrm{K}_{5}$ & $\mathrm{~K}_{6}$ & $\mathrm{~K}_{7}$ & $\mathrm{~K}_{8}$ & $\mathrm{~K}_{9}$ & $\mathrm{~K}_{10}$ \\
\hline 1\{ & $\begin{array}{l}\mathrm{ma} \\
\mathrm{mi}\end{array}$ & $\begin{array}{l}0.41 \\
0.20\end{array}$ & $\begin{array}{r}-0.25 \\
0.25\end{array}$ & $\begin{array}{l}0.88 \\
0.75\end{array}$ & $\begin{array}{r}-0.02 \\
0.06\end{array}$ & $\begin{array}{l}5.82 \\
5.82\end{array}$ & $\begin{array}{l}4.67 \\
4.67\end{array}$ & $\begin{array}{l}2.70 \\
2.70\end{array}$ & $\begin{array}{l}2.17 \\
2.17\end{array}$ & $\begin{array}{l}1.15 \\
1.15\end{array}$ & $\begin{array}{l}0.53 \\
0.53\end{array}$ \\
\hline 3\{ & $\begin{array}{l}\mathrm{ma} \\
\mathrm{mi}\end{array}$ & $\begin{array}{l}0.21 \\
0.14\end{array}$ & $\begin{array}{l}0.53 \\
0.52\end{array}$ & $\begin{array}{l}0.79 \\
0.77\end{array}$ & $\begin{array}{l}0.05 \\
0.07\end{array}$ & $\begin{array}{l}5.97 \\
5.78\end{array}$ & $\begin{array}{l}4.21 \\
4.88\end{array}$ & $\begin{array}{l}2.79 \\
2.60\end{array}$ & $\begin{array}{l}1.71 \\
2.38\end{array}$ & $\begin{array}{l}1.76 \\
0.90\end{array}$ & $\begin{array}{l}1.08 \\
0.22\end{array}$ \\
\hline 4\{ & $\begin{array}{l}\mathrm{ma} \\
\mathrm{mi}\end{array}$ & $\begin{array}{l}0.41 \\
0.39\end{array}$ & $\begin{array}{l}-0.35 \\
-0.36\end{array}$ & $\begin{array}{l}0.83 \\
0.82\end{array}$ & $\begin{array}{l}-0.03 \\
-0.03\end{array}$ & $\begin{array}{l}5.78 \\
6.03\end{array}$ & $\begin{array}{l}4.91 \\
4.23\end{array}$ & $\begin{array}{l}2.56 \\
2.89\end{array}$ & $\begin{array}{l}2.41 \\
1.73\end{array}$ & $\begin{array}{l}0.87 \\
1.80\end{array}$ & $\begin{array}{l}0.15 \\
1.16\end{array}$ \\
\hline 5\{ & $\begin{array}{l}\mathrm{ma} \\
\mathrm{mi}\end{array}$ & $\begin{array}{l}0.37 \\
0.18\end{array}$ & $\begin{array}{r}-0.31 \\
0.24\end{array}$ & $\begin{array}{l}0.88 \\
0.76\end{array}$ & $\begin{array}{r}-0.04 \\
0.04\end{array}$ & $\begin{array}{l}5.86 \\
5.92\end{array}$ & $\begin{array}{l}4.80 \\
4.13\end{array}$ & $\begin{array}{l}2.71 \\
2.68\end{array}$ & $\begin{array}{l}2.29 \\
1.62\end{array}$ & $\begin{array}{l}1.06 \\
1.79\end{array}$ & $\begin{array}{l}0.42 \\
1.06\end{array}$ \\
\hline 7 & & 0.25 & 0.49 & 0.79 & 0.04 & 5.91 & 4.14 & 2.68 & 1.64 & 1.77 & 1.04 \\
\hline 10\{ & $\begin{array}{l}\mathrm{ma} \\
\mathrm{mi}\end{array}$ & $\begin{array}{l}0.39 \\
0.17\end{array}$ & $\begin{array}{r}-0.28 \\
0.38\end{array}$ & $\begin{array}{l}0.85 \\
0.74\end{array}$ & $\begin{array}{r}-0.04 \\
0.03\end{array}$ & $\begin{array}{l}5.79 \\
5.76\end{array}$ & $\begin{array}{l}4.96 \\
5.00\end{array}$ & $\begin{array}{l}2.51 \\
2.49\end{array}$ & $\begin{array}{l}2.45 \\
2.50\end{array}$ & $\begin{array}{l}0.83 \\
0.77\end{array}$ & $\begin{array}{r}0.06 \\
-0.01\end{array}$ \\
\hline 11\{ & $\begin{array}{l}\mathrm{ma} \\
\mathrm{mi}\end{array}$ & $\begin{array}{l}0.25 \\
0.25\end{array}$ & $\begin{array}{l}0.14 \\
0.15\end{array}$ & $\begin{array}{l}0.82 \\
0.81\end{array}$ & $\begin{array}{l}0.03 \\
0.02\end{array}$ & $\begin{array}{l}5.99 \\
5.84\end{array}$ & $\begin{array}{l}4.22 \\
4.76\end{array}$ & $\begin{array}{l}2.80 \\
2.65\end{array}$ & $\begin{array}{l}1.72 \\
2.26\end{array}$ & $\begin{array}{l}1.77 \\
1.08\end{array}$ & $\begin{array}{l}1.08 \\
0.39\end{array}$ \\
\hline 16\{ & & $\begin{array}{l}0.35 \\
0.25\end{array}$ & $\begin{array}{r}-0.20 \\
0.22\end{array}$ & $\begin{array}{l}0.87 \\
0.80\end{array}$ & $\begin{array}{r}-0.02 \\
0.02\end{array}$ & $\begin{array}{l}5.97 \\
5.86\end{array}$ & $\begin{array}{l}4.30 \\
4.71\end{array}$ & $\begin{array}{l}2.82 \\
2.71\end{array}$ & $\begin{array}{l}1.80 \\
2.21\end{array}$ & $\begin{array}{l}1.67 \\
1.15\end{array}$ & $\begin{array}{l}1.02 \\
0.50\end{array}$ \\
\hline 17\{ & & $\begin{array}{l}0.16 \\
0.23\end{array}$ & $\begin{array}{l}0.30 \\
0.24\end{array}$ & $\begin{array}{l}0.83 \\
0.83\end{array}$ & $\begin{array}{r}0.02 \\
-0.03\end{array}$ & $\begin{array}{l}5.88 \\
5.92\end{array}$ & $\begin{array}{l}4.86 \\
4.75\end{array}$ & $\begin{array}{l}2.75 \\
2.78\end{array}$ & $\begin{array}{l}2.33 \\
2.24\end{array}$ & $\begin{array}{l}1.05 \\
1.17\end{array}$ & $\begin{array}{l}0.42 \\
0.54\end{array}$ \\
\hline 19\{ & & $\begin{array}{l}0.27 \\
0.29\end{array}$ & $\begin{array}{l}0.35 \\
0.22\end{array}$ & $\begin{array}{l}0.71 \\
0.72\end{array}$ & $\begin{array}{l}0.01 \\
0.00\end{array}$ & $\begin{array}{l}5.71 \\
5.75\end{array}$ & $\begin{array}{l}4.81 \\
4.71\end{array}$ & $\begin{array}{l}2.50 \\
2.54\end{array}$ & $\begin{array}{l}2.31 \\
2.20\end{array}$ & $\begin{array}{l}0.90 \\
1.04\end{array}$ & $\begin{array}{l}0.20 \\
0.34\end{array}$ \\
\hline 20 & & 0.24 & 0.29 & 0.69 & 0.04 & 5.70 & 4.80 & 2.52 & 2.29 & 0.90 & 0.23 \\
\hline 22\{ & & $\begin{array}{l}0.53 \\
0.37\end{array}$ & $\begin{array}{r}-0.27 \\
0.29\end{array}$ & $\begin{array}{l}0.87 \\
0.82\end{array}$ & $\begin{array}{l}-0.03 \\
-0.00\end{array}$ & $\begin{array}{l}6.10 \\
5.91\end{array}$ & $\begin{array}{l}4.17 \\
4.71\end{array}$ & $\begin{array}{l}2.86 \\
2.67\end{array}$ & $\begin{array}{l}1.67 \\
2.21\end{array}$ & $\begin{array}{l}1.93 \\
1.20\end{array}$ & $\begin{array}{l}1.19 \\
0.46\end{array}$ \\
\hline 24\{ & $\begin{array}{l}\mathrm{ma} \\
\mathrm{mi}\end{array}$ & $\begin{array}{l}0.05 \\
0.16\end{array}$ & $\begin{array}{l}0.60 \\
0.49\end{array}$ & $\begin{array}{l}0.73 \\
0.74\end{array}$ & $\begin{array}{l}0.13 \\
0.09\end{array}$ & $\begin{array}{l}5.65 \\
5.75\end{array}$ & $\begin{array}{l}5.08 \\
4.96\end{array}$ & $\begin{array}{l}2.47 \\
2.56\end{array}$ & $\begin{array}{l}2.58 \\
2.46\end{array}$ & $\begin{array}{l}0.57 \\
0.79\end{array}$ & $\begin{array}{r}-0.11 \\
0.11\end{array}$ \\
\hline 30\{ & $\begin{array}{l}\mathrm{ma} \\
\mathrm{mi}\end{array}$ & $\begin{array}{l}0.12 \\
0.24\end{array}$ & $\begin{array}{l}0.20 \\
0.17\end{array}$ & $\begin{array}{l}0.78 \\
0.77\end{array}$ & $\begin{array}{r}0.04 \\
-0.00\end{array}$ & $\begin{array}{l}5.77 \\
5.80\end{array}$ & $\begin{array}{l}4.82 \\
4.70\end{array}$ & $\begin{array}{l}2.65 \\
2.68\end{array}$ & $\begin{array}{l}2.32 \\
2.20\end{array}$ & $\begin{array}{l}0.95 \\
1.10\end{array}$ & $\begin{array}{l}0.33 \\
0.48\end{array}$ \\
\hline 32 & & 0.21 & 0.17 & 0.75 & 0.02 & 5.75 & 4.73 & 2.63 & 2.22 & 1.02 & 0.41 \\
\hline 35 & & 0.21 & 0.20 & 0.77 & 0.00 & 5.77 & 4.75 & 2.67 & 2.24 & 1.02 & 0.43 \\
\hline 38 & & 0.17 & 0.39 & 0.79 & 0.04 & 5.81 & 4.90 & 2.66 & 2.40 & 0.91 & 0.27 \\
\hline 96\{ & $\begin{array}{l}\mathrm{ma} \\
\mathrm{mi}\end{array}$ & $\begin{array}{r}-0.19 \\
0.30\end{array}$ & $\begin{array}{l}0.95 \\
0.21\end{array}$ & $\begin{array}{l}0.53 \\
0.63\end{array}$ & $\begin{array}{r}0.07 \\
-0.06\end{array}$ & $\begin{array}{l}5.32 \\
5.83\end{array}$ & $\begin{array}{l}5.43 \\
4.67\end{array}$ & $\begin{array}{l}2.00 \\
2.51\end{array}$ & $\begin{array}{l}2.93 \\
2.16\end{array}$ & $\begin{array}{r}-0.12 \\
1.17\end{array}$ & $\begin{array}{r}-0.93 \\
0.35\end{array}$ \\
\hline 99\{ & $\begin{array}{l}\mathrm{ma} \\
\mathrm{mi}\end{array}$ & $\begin{array}{r}-0.44 \\
0.32\end{array}$ & $\begin{array}{l}1.13 \\
0.11\end{array}$ & $\begin{array}{l}0.56 \\
0.78\end{array}$ & $\begin{array}{l}0.23 \\
0.02\end{array}$ & $\begin{array}{l}5.02 \\
5.79\end{array}$ & $\begin{array}{l}5.64 \\
4.61\end{array}$ & $\begin{array}{l}1.87 \\
2.63\end{array}$ & $\begin{array}{l}3.14 \\
2.10\end{array}$ & $\begin{array}{r}-0.62 \\
1.81\end{array}$ & $\begin{array}{r}-1.27 \\
0.53\end{array}$ \\
\hline $101 \mathrm{~A}$ & & 0.04 & 0.93 & 0.84 & 0.06 & 5.68 & 5.47 & 2.62 & 2.97 & 0.21 & -0.35 \\
\hline $101 \mathrm{~B}$ & & 0.31 & 0.01 & 0.67 & 0.02 & 5.81 & 4.53 & 2.63 & 2.02 & 1.28 & 0.61 \\
\hline 104\{ & $\begin{array}{l}\mathrm{ma} \\
\mathrm{mi}\end{array}$ & $\begin{array}{l}0.23 \\
0.24\end{array}$ & $\begin{array}{l}-0.09 \\
-0.21\end{array}$ & $\begin{array}{l}0.81 \\
0.80\end{array}$ & $\begin{array}{l}-0.04 \\
-0.06\end{array}$ & $\begin{array}{l}5.80 \\
5.82\end{array}$ & $\begin{array}{l}4.55 \\
4.38\end{array}$ & $\begin{array}{l}2.71 \\
2.73\end{array}$ & $\begin{array}{l}2.05 \\
1.88\end{array}$ & $\begin{array}{l}1.24 \\
1.44\end{array}$ & $\begin{array}{l}0.66 \\
0.86\end{array}$ \\
\hline
\end{tabular}

regression line should be equal to $K_{D}=1.0$ at equilibrium conditions

If these suggestions are correct, then it follows that $\mathrm{K}^{\mathrm{cpx} / \mathrm{liq}} \mathrm{D}(\mathrm{Fe} / \mathrm{Mg})$ (Fig. 9) also is independent of temperature but varies as a function of composition. The plotting on the olivine temperature- log $(\mathrm{Fe} / \mathrm{Mg})$ in clinopyroxene diagram (Fig. 10) shows linear cor- 


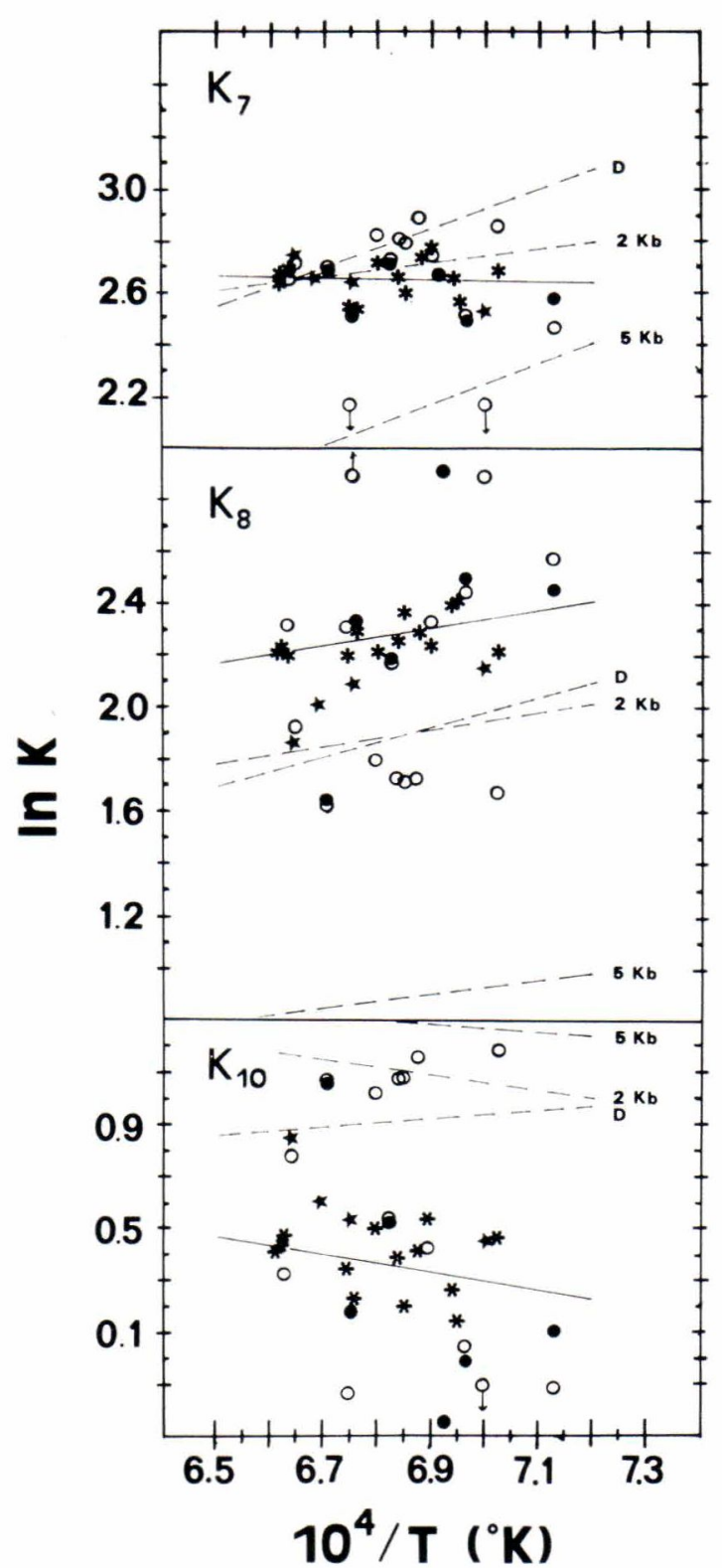

Fig. 7. Effect of pressure on equilibrium constants $\mathrm{K}_{7}, \mathrm{~K}_{8}$ and $\mathrm{K}_{10}$ (see text). Symbols and lines are the same as in Fig. 6.

relation between these parameters. However because the $\mathrm{Fe} / \mathrm{Mg}$ in liquid correlates well with the derived temperatures (Fig. 11) this might cause the correlation between the $\mathrm{Fe} / \mathrm{Mg}$ in clinopyroxene and temperature.

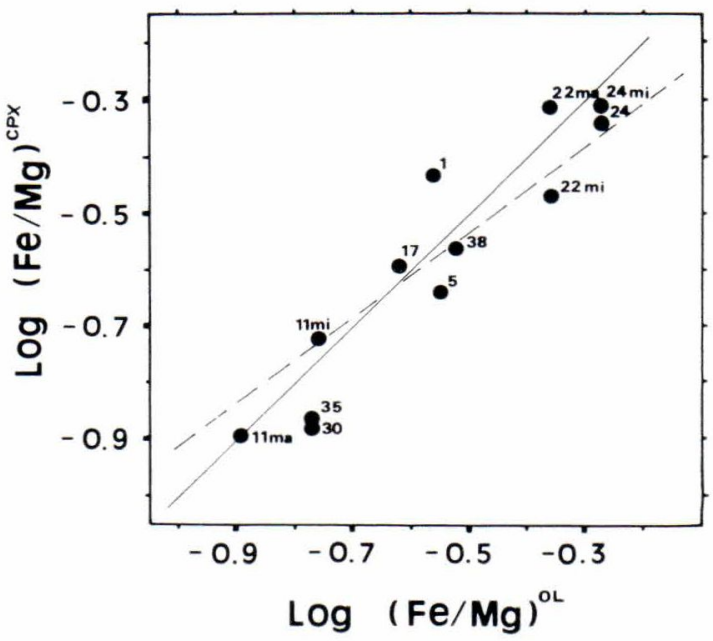

Fig. 8. Log $(\mathrm{Fe} / \mathrm{Mg})$ in olivine plotted against $\log$ $(\mathrm{Fe} / \mathrm{Mg})$ in clinopyroxene. Dashed line indicates equilibrium in Duke's (1976) experiments; solid line $\left(\mathrm{K}_{\mathrm{D}}=1.0\right)$ in this study. Numbers refer to the samples (Mäkipää 1978).

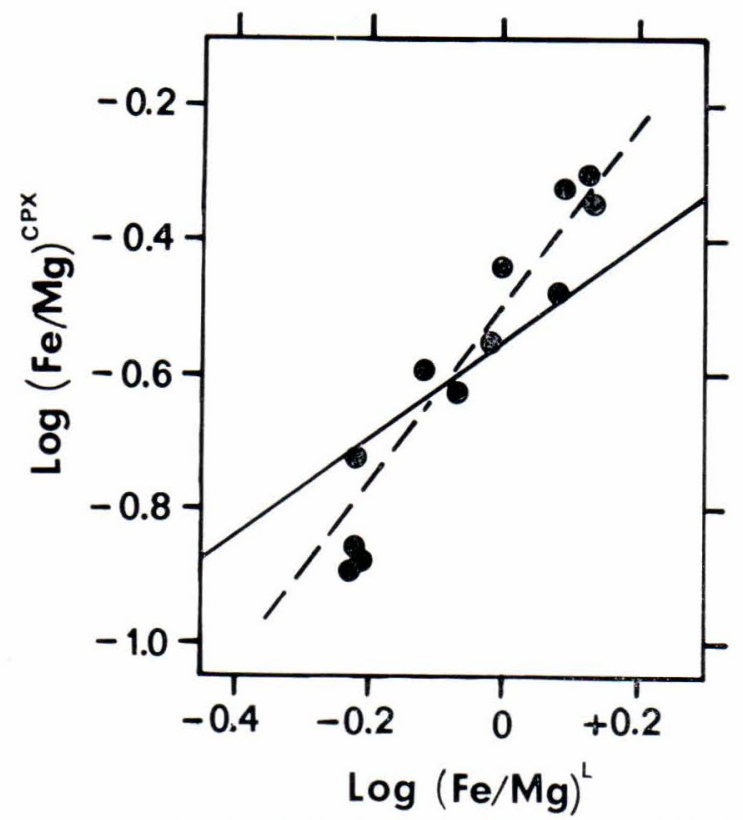

Fig. 9. Log (Fe/Mg) in clinopyroxene plotted against $\log (\mathrm{Fe} / \mathrm{Mg})$ in liquid. Solid line indicates equilibrium in Duke's (1976) experiments, dashed line in this study.

Powell and Powell (1974) formulated a geothermometer based on a calculated temperature dependence on the distribution of 

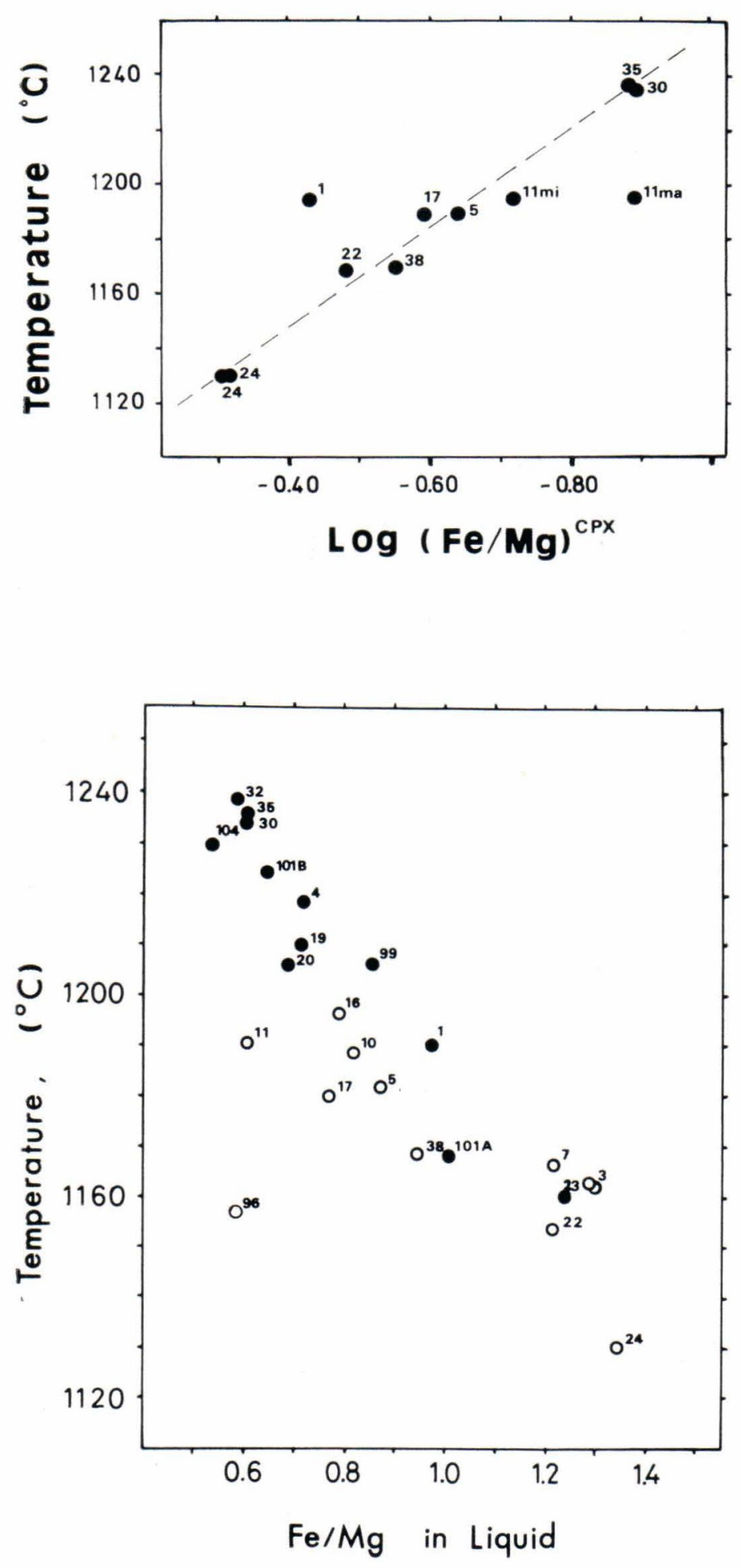

Fig. 10. Log (Fe/Mg) in clinopyroxene plotted against calculated equilibrium temperature (Table 3). Numbers refer to the samples (Mäkipää 1978).
Fig. 11. The $\mathrm{Fe} / \mathrm{Mg}$ in liquid plotted against calculated equilibrium temperature (Table 3 ). $\bullet=$ olivine normative tholeiite $\mathrm{O}=$ quartz normative tholeiite. Numbers refer to the samples (Mäkipää 1978). 
iron and magnesium between olivine and calcic pyroxene. Their model requires that the pressure is known which sets a limit to its use in this study. Wood (1976) concluded that the Powell and Powell geothermometer is not applicable in its present form, because it is independent of olivine and clinopyroxene compositions but depends in fact only on the aluminium content in clinopyroxene.

All other clinopyroxene geothermometers are inapplicable in the present study because they require garnet or a spinel phase to be present and olivine and plagioclase absent.

\section{Discussion}

\section{Mineral equilibrium}

In the original hyaloclastites the glass phase as well as the phenocrysts were found to be homogenous both in major and trace elements (Mäkipää 1978). Using various equilibrium tests all microphenocrysts are in equilibrium, but some macrophenocrysts are clearly out of equilibrium.

The most successful test for olivine equilibrium is the distribution of $\mathrm{Mg}$ and $\mathrm{Fe}$ between crystal and liquid. The role of ferric iron in liquid must be evaluated before attempting the distribution coefficient $\mathrm{K}_{\mathrm{D}}$ to natural rocks. The average $\mathrm{K}_{\mathrm{D}}(0.299)$ agrees well with the Roeder and Emslie value of $\mathrm{K}_{\mathrm{D}}=0.30$.

Drake $(1972,1975)$ suggested the use of major element distribution between plagioclase and liquid versus temperature for an equilibrium test. Using the present experimental results for Icelandic tholeiites the sodium distribution is the most successful test for equilibrium.

The clinopyroxene equilibrium test also is complicated because it depends on major element composition, pressure and temperature. However, the most successful test for clinopyroxene equilibrium is the distribution of $\mathrm{Fe}$ and $\mathrm{Mg}$ between equilibrium olivines and clinopyroxene.

\section{Geothermometers}

The crystallization temperature or the temperature of the magma on eruption can be calculated from the mineral-liquid analyses of the hyaloclastites. The calculated temperatures here using the various methods range from 1130 to $1238^{\circ} \mathrm{C}$ which are within the crystallization range of basalt measured in experiments of several authors.

The most successful of these geothermometers is the olivine-liquid combination (Roeder and Emslie 1970). Roeder (1974) modified his earlier results and the temperature can be calculated from $\mathrm{MgO}, \mathrm{FeO}$ and $\mathrm{MnO}$ distributions.

The MgO distribution is, however, most useful, because the temperature derived from the $\mathrm{FeO}$ distribution is dependent on the oxidation state of the magma which is uncertain and to derive temperature from the $\mathrm{MnO}$ distribution special determinations are needed.

The plagioclase temperature estimates are more complicated because the elemental distribution is affected by water pressure. Kudo and Weill (1970) formulated a geothermometer which Mathez (1973) modified. The use of the Mathez modification gives results which are in close agreement with experimental data and calculated olivine temperatures. It is noticeable that using Mathez modification the calculated temperatures have a maximum at $\mathrm{PH}_{2} \mathrm{O}=0.5 \mathrm{~kb}$ and then decrease again. Fig. 12 shows schematically the rising water pressure influence on the calculated temperatures.

The odd part about this diagram is the maximum temperature. This might be due to the incorrect assumption by Mathez (1973) 


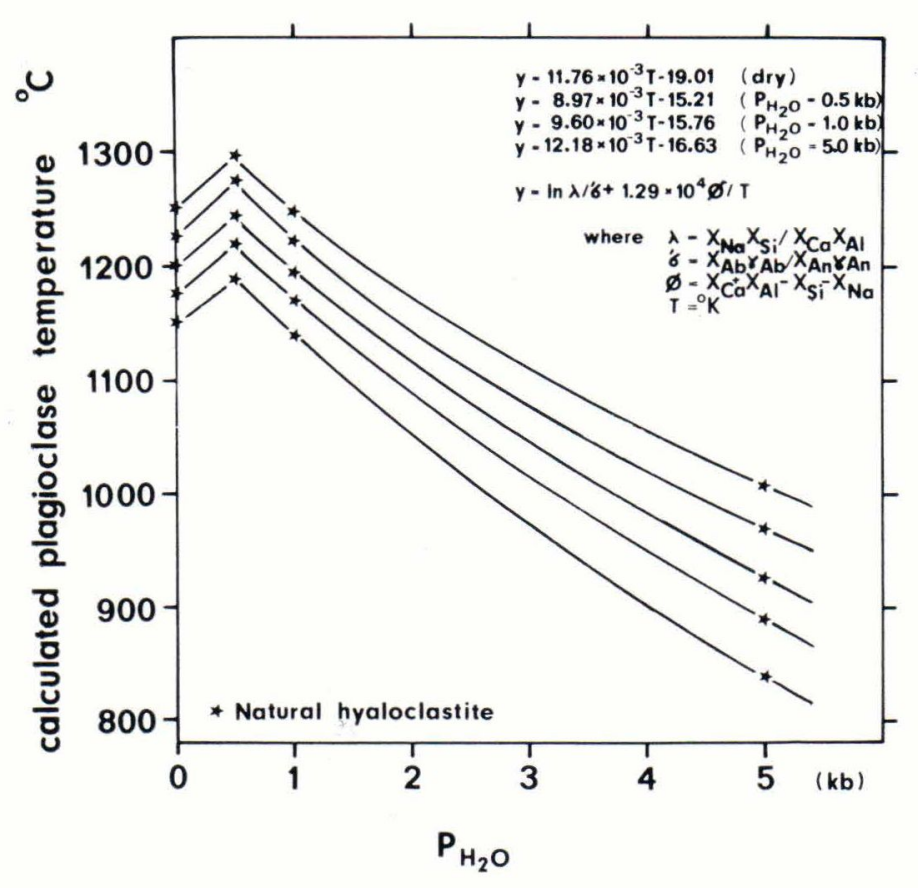

Fig. 12. Effect of water pressure on the calculated plagioclase temperatures. Equations: (dry) - Mathez 1973 (Eq. 7a), (0.5kb) — Mathez 1973 (Eq. 7.b), (1.0kb) - Mathez 1973 (Eq. 7c), (5.0kb) - Kudo and Weill 1970. that neither the temperature nor water pressure significantly affect $\gamma_{\mathrm{Ab}} / \gamma_{\mathrm{An}}$ or else that the effects of these variables oppose each other.

The role of the first crystallization phase

The geothermometers available give equilibrium temperatures which can be substantially lower than the liquidus temperatures. However, in view of the very slight crystallization (usually about $5 \%$ ) of the samples the calculated temperatures must be very near the liquidus temperatures. Accepting this there is a complication in these calculations because they depend on the first crystallization phase.

The amount of the crystals present needs not indicate the first phase and the best indicator is the relation of the various crystal phases to each other. Careful observations of the intercrystal relationships showed that either plagioclase or olivine could be the first phase to crystallize. In some cases one phase was closely followed by the other or they may have coprecipitated.

If olivine is the first phase to crystallize from a liquid then the calculated olivine temperatures are liquidus temperatures. This, however, leads to $\mathrm{Ca}, \mathrm{Na}$ and $\mathrm{Al}$ increase in the liquid and the plagioclase temperatures derived are too high. If plagioclase crystallizes first then both $\mathrm{Mg}$ and $\mathrm{Fe}$ in the liquid will increase. This increase depends on the liquid composition and how much plagioclase crystallizes i.e. how far the composition is from the contecticum. Therefore the early crystallization of plagioclase will increase the apparent temperature of the crystallization and it will not affect the composition of the crystallizing phases.

However this increase is not very marked in the present study, because of the slight crystallization. With solidification of $20 \%$ the temperature difference using the $\mathrm{FeO}^{\mathrm{l}}$ $\mathrm{MgO}^{1}$ temperatures (Fig. 2) is about $60^{\circ} \mathrm{C}$ and 
Fig. 13. The change of the measured

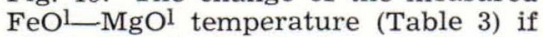
plagioclase is the first crystallization phase $(\%$ by volume). A 71108 Lava from Drekagil, Dyngjufjöll. Nal 27 - Pillow lava from Leirhafnarfjall, Melrakksletta. VE 67 - Lava from Helgafell, Westman Islands. Surtsey - The first lava in the Surtsey eruption (1963-1967). H. A. Ljosu - Lava near Ljosaskrida, Snæfellsnes. 16 - Vifilsfell (RE-16; Mäkipää 1978). 30 - Hestfjall (HE-30; Mäkipää 1978). Solid line is the best fitting by method of least squares. $\mathrm{R}=$ correlation coefficient.

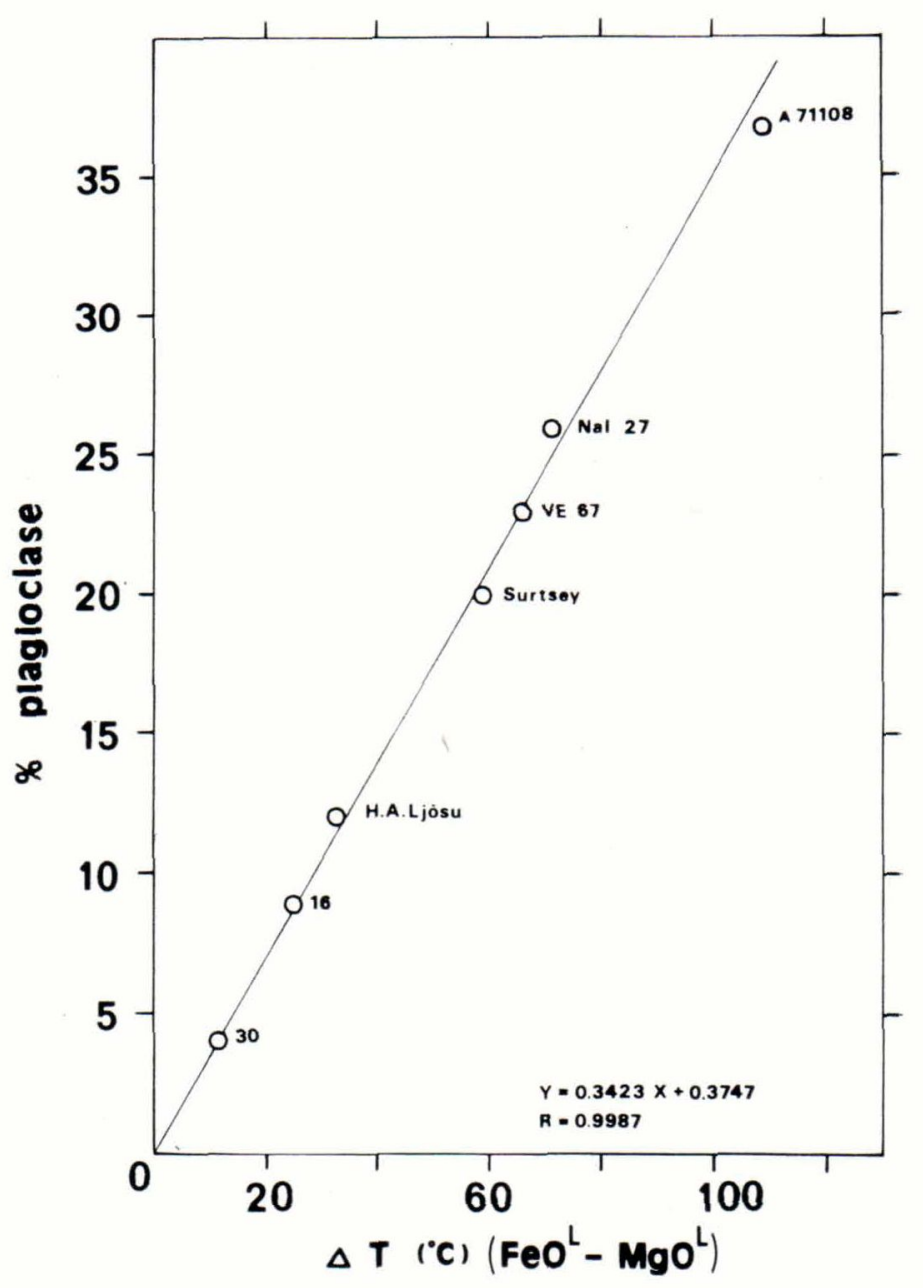

increases regularly with increasing solidification (Fig. 13). In some samples used it is estimated that less than $5 \%$ of plagioclase crystallized before olivine started to crystallize. This means that the maximum temperature difference for these samples would be about $10-20^{\circ} \mathrm{C}$.

The Ol-Di-Pl diagram (Fig. 14) shows the possible first crystallization phase in the individual samples. Shibata (1976) divided the Ol-Di-Pl diagram to two major areas, olivine and plagioclase fields. Shibata's division line goes very near that dashed line which makes the difference between plagioclase and pla- gioclase +olivine fields in the present study. The division between olivine and plagioclase +olivine fields is shown.

\section{Model of the fractional crystallization}

As mentioned earlier the calculated temperatures must be very near the liquidus temperatures of these magmas. Therefore it is of particular interest to compare these 'liquidus' temperatures with the simple fractional crystallization curve measured in Hawaiian basalts (Yoder and Tilley 1962). On the A'F'M diagram (Fig. 15) the present data 

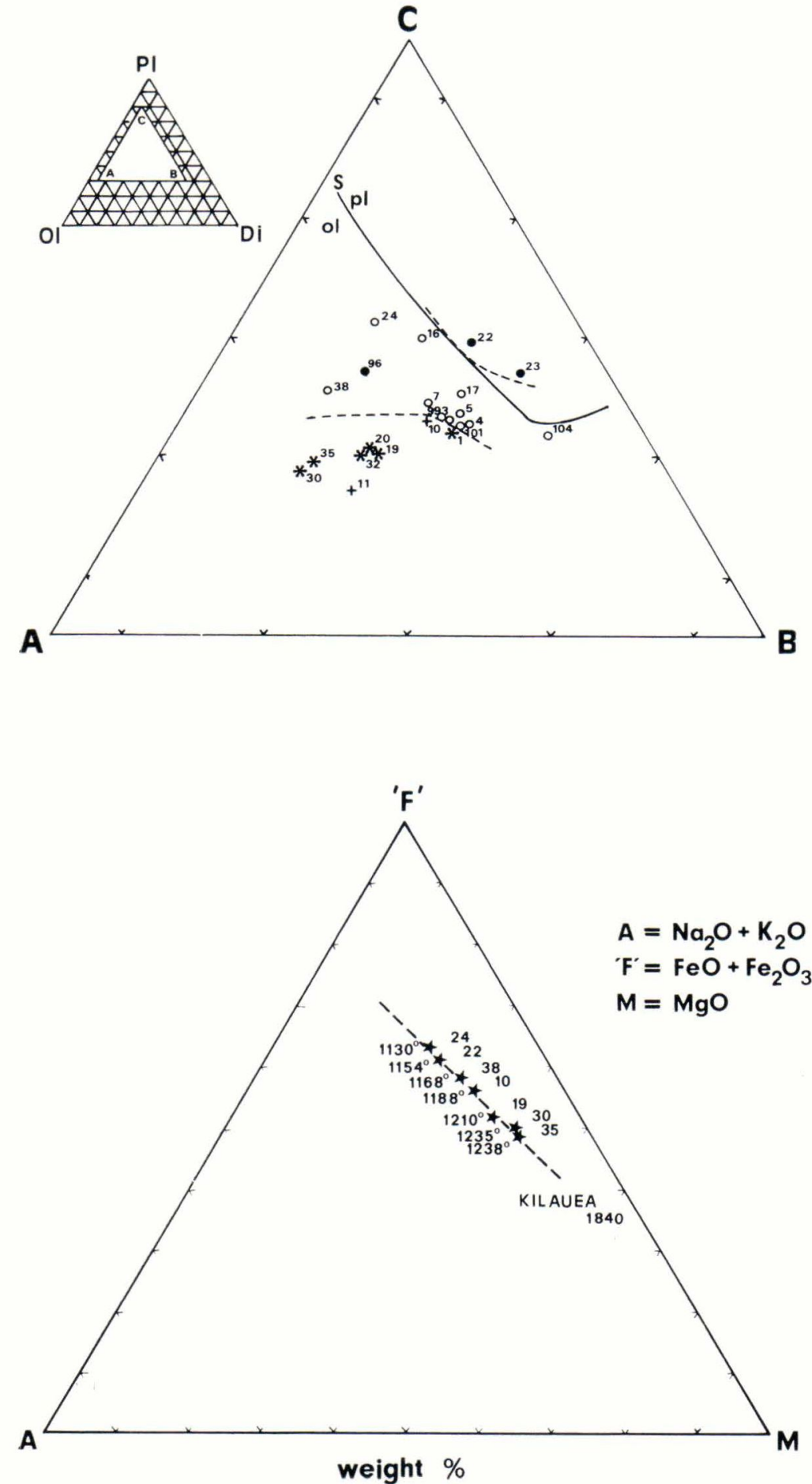

Fig. 14. Plot of the analyses (Mäkipää 1978. Table 1) on the Di-Ol-Pl diagram in molecular proportions. $\mathrm{Di}=\mathrm{Ca}(\mathrm{Mg}$, $\mathrm{Fe}) \mathrm{Si}_{2} \mathrm{O}_{6} ; \mathrm{Ol}=(\mathrm{Mg}, \mathrm{Fe})_{2} \mathrm{SiO}_{4} ; \mathrm{Pl}$ $=\mathrm{CaAl}_{2} \mathrm{Si}_{2} \mathrm{O}_{8}+\mathrm{NaAlSi}_{3} 0_{8}$. The first phase to crystallize from the basaltic melt is: $\mathbb{*}+$ olivine $\bigcirc$ - plagioclase + olivine - plagioclase. Solid line (S) separates the plagioclase field (pl) and olivine field (ol) in Shibata's (1976) investigation. Dashed lines separate the phase fields in this study. Numbers refer to the samples (Mäkipää 1978).

Fig. 15. A'F'M plot of some hyaloclastites with their "silicate liquidus temperatures». Dashed line is the trend of the 1840 flow of Kilauea (Yoder and Tilley 1962, Fig. 8). Numbers refer to the samples (Mäkipää 1978). 
shows a trend which is identical with the line formed by the simple fractional crystallization in Hawaiian basalts. On the basis of this diagram the calculated temperatures are in excellent agreement with the experimental data of Yoder and Tilley (1962).

The temperature $-\mathrm{FeO}^{\mathrm{t}} /\left(\mathrm{FeO}^{\mathrm{t}}+\mathrm{MgO}\right)$ relation has been shown to be linear for Hawaiian basalts (Tilley et al. 1964). As seen in Fig. 16 all the calculated temperatures are higher than those measured experimentally by Tilley et al. (1964). This difference (average $18^{\circ} \mathrm{C}$ ) is most likely due to different composition and oxidation state in Icelandic basalts as well as the difference in experimental procedures. Therefore it is interesting to note that those Icelandic basalts (Eldgjá lava) used in Tilley's experiments fall exactly on the line presented here for Icelandic hyaloclastites.

If we compare the present experimentally determined liquidus temperatures (symbol+ in Fig. 16) and calculated hyaloclastite temperatures the agreement is excellent.

\section{Geobarometers}

The derived plagioclase temperatures are found to vary with water pressures. This offers a possibility to estimate the prevailing water pressure during the crystallization.

Before such estimates can be derived some adjustments must be done. From the Fig. 5 and Table 4 it emerges that samples TH-3, TH-4, RE-11, KE-24 and TJ-104 are slightly out of equilibrium. If the water pressure is derived then it must be done on the basis of equilibrium crystals. To reach the equilibrium the composition of these plagioclases must be changed. This means that the calculated temperature in the sample $\mathrm{TH}-3$ rises about $30-40^{\circ} \mathrm{C}$ The corresponding value for $\mathrm{KE}-24$ is about $20-30^{\circ} \mathrm{C}$. Contrary to the above the temperatures are lower by 10 -

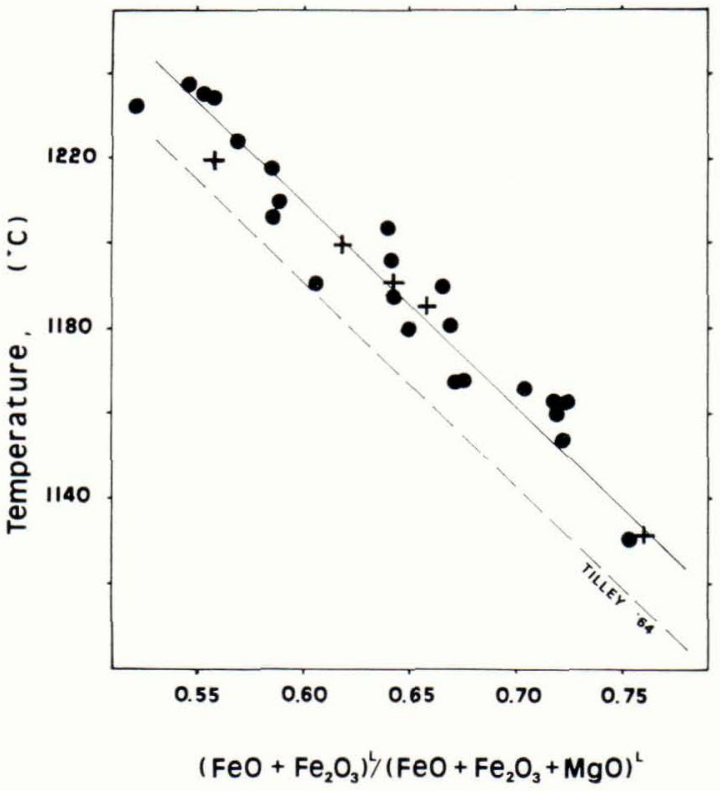

Fig. 16. Plot correlating »liquidus temperatures» with iron enrichment in hyaloclastites. Dashed line drawn after Tilley et al. (1964). $=$ hyaloclastite, $+=$ present experiment data. Solid line is the best fitting by method of least squares to hyaloclastite data only.

$20^{\circ} \mathrm{C}, 40-50^{\circ} \mathrm{C}$ and $20-30^{\circ} \mathrm{C}$ in RE- 11 , TH- 4 and TJ 104, respectively.

In Fig. 17 the calculated temperatures are compared on the basis of different water pressures using temperatures derived from equilibrium olivines and plagioclases. In an earlier paper (Mäkipää 1978) it was concluded on the basis of CMAS- and Ab-An-Di-Fo systems that individual samples represent different water pressures (about 0, 1 and 2 $\mathrm{kb})$. The agreement with the present data is good.

The temperatures calculated for the present $1 \mathrm{~atm}$ pressure experiments agree well with the Mathez (1973) $0 \mathrm{~kb}$ temperature.

The experimental results show that the $\mathrm{fO}_{2}$ affects the coexisting phases. In natural basalts the $\mathrm{fO}_{2}$ follows the QMF-buffer. This gives a value of $\log \mathrm{fO}_{2}$ at $1200^{\circ} \mathrm{C}$ of about -8.3. It is well known that the $\mathrm{fO}_{2}$ affects the 


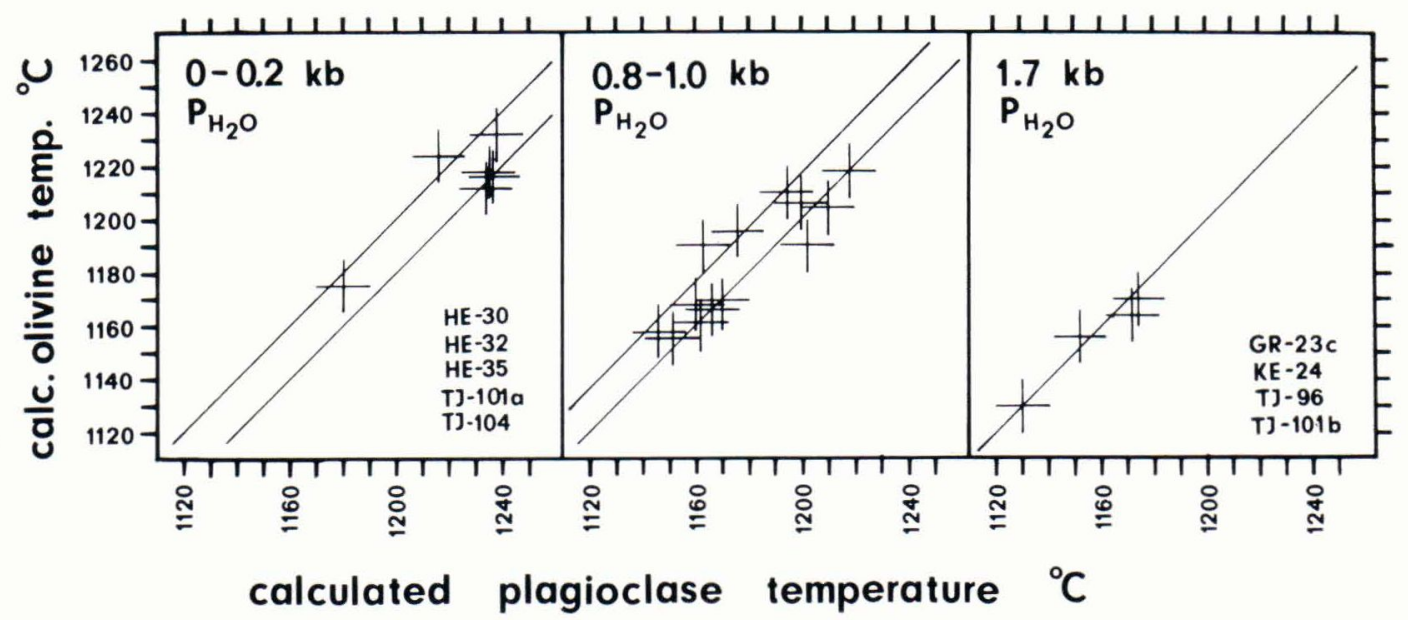

Fig. 17. Comparison of calculated olivine equilibrium temperatures as a function of calculated plagioclase temperatures on the basis of different water pressures. Numbers refer to the samples (Mäkipää 1978).

olivine composition (Roeder and Emslie, 1970). This can easily be seen also in the present experiments where the olivine composition change is only $2 \%$ forsterite although the temperature difference should indicate much greater variation in forsterite content (see Table 1).

Although the $\mathrm{fO}_{2}$ in some experimental runs (KRA-731) were as low as $\log \mathrm{fO}_{2}=$ -11 this has not seriously affected the calculated plagioclase temperatures. This indicates that water pressure has much greater influence on the calculated equilibrium temperatures than oxygen fugacity. Further experiments will be carried out using direct measurements in furnace to evaluate the effect of the oxygen fugacity. The solubility of water in magmas is known to be dependent on volatile contents of magma and pressure conditions. In a view of the small number of estimates of the water content of the original magma using glass inclusion chemistry (Anderson 1973), it appears that those basalts which have the lowest estimated water pressure also have the lowest original water content.
Accepting the assumption that the calculated temperatures are close to the liquidus temperatures, the use of experimental results by Yoder and Tilley (1962, Fig. 34) in natural olivine tholeiite-water systems, shows that during the crystallization of these hyaloclastites the total pressure was greater than the water pressure.

In conclusion it can be said that a combination of actual melting experiments and the use of published mineral-liquid equilibrium studies can at the present be usefully employed to estimate various properties of the system.

However, there is still some serious discrepancies and the results must be interpreted with great care. However, valid indications about equilibrium, temperature and possibly water pressure can be derived.

Acknowledgements - This study was carried out during the authors fellowship at the Nordic Volcanological Institute, Reykjavik. Drs. Gudmundur E. Sigvaldason, Karl Grönvold and K. Korsman kindly read the manuscript and provided valuable suggestions; this is gratefully acknowledged. 


\section{References}

Anderson, A. T. (1973) The before-eruption water content of some high-alumina magmas. Bull. Volcan. 37: $530-553$.

Bottinga, Y., Kudo, A. and Weill, P. (1966) Some observations on oscillatory zoning and crystallization of magmatic plagioclase. Am. Miner. 51: $792-806$.

Bowen, N. L. (1913) The melting phenomena of the plagioclase feldspars. Am. J. Sci., 4th Ser. 35: $577-599$.

- (1915) The crystallization of haplobasaltic, haplodioritic and related magmas. Am. J. Sci., 4th Ser. 40: 161-185.

- and Schairer, J. F. (1935) The system MgOFeO-SiO2. Am. J. Sci. 29: 151-217.

Bradley, R. S. (1962) Thermodynamic calculations on phase equilibria involving fused salts. Part II. Solid solutions and application to the olivines. Am. J. Sci. 260: 550-554.

Carmichael, I. S. E. (1960) The feldspar phenocryst of some Tertiary acid glasses. Min. Mag. 32 : $587-608$.

Drake, M. J. (1972) The distribution of major and trace elements between plagioclase feldspar and magmatic silicate liquid: an experimental study. Ph. D. Dissertation University of Oregon, Eugene, Ore.

- (1975) Plagioclase thermometry and barometry in igneous systems. In Extended Abstracts: International Conference on Geothermometry and Geobarometry, Pennsylvania State University, Pennsylvania.

- (1976) Plagioclase-melt equilibria. Geochim. Cosmochim. Acta 40: 457-465.

- and Weill, D. F. (1975) Partition of Sr, Ba, Ca, $\mathrm{Y}, \mathrm{Eu}^{2+}, \mathrm{Eu}^{3+}$, and other REE between plagioclase feldspar and magmatic liquid: an experimental study. Geochim. Cosmochim. Acta 39: $689-712$.

Duke, J. M. (1976) Distribution of the period four transition elements among olivine, calcic clinopyroxene and mafic silicate liquid: experimental results. J. Petrol. 17: 499-521.

Kennedy, G. C. (1948) Equilibrium between volatiles and iron oxides in igneous rocks. Am. J. Sci. 246: $529-549$.

Kudo, A. M. and Weill, D. F. (1970) An igneous plagioclase geothermometer. Contr. Mineral. Petrol. 25: 52-65.

Larsen, E. S. and Irving, J. (1938) Petrologic results of the mineral from the Tertiary volcanic rocks of the San Juan region, Color- ado. 7. The plagioclase feldspars. Am. Miner. 23: $227-257$.

Leeman, W. P. and Scheidegger, K. F. (1977) Olivine/liquid distribution coefficients and a test for crystal-liquid equilibrium. Earth Planet. Sci. Lett. 35: 247-257.

Longhi, J., Walker, D. and Hays, J. F. (1975) Fe$\mathrm{Mg}$ distribution between olivine and lunar basaltic liquids. EOS (Am. Geophys. Union Trans.) 56: 471.

Mäkipää, H. T. (1978) Petrological relations in some Icelandic basaltic hyaloclastites. Bull. Geol. Soc. Finland 50:81-112.

Mathez, E. A. (1973) Refinement of the KudoWeill plagioclase geothermometry and its application to basaltic rocks. Contr. Mineral. Petrol. 41: 61-72.

Mysen, B. O. and Boettcher, A. L. (1975) Experimentally determined pressure, temperature and chemical variables in peridotites to 30 kbar: geothermometry and geobarometry. In Extended Abstracts: International Conference on Geothermometry and Geobarometry, Pennsylvania State University, Pennsylvania.

Orville, P. M. (1972) Plagioclase cation exchange equilibria with aqueous chloride solution: results at $700{ }^{\circ} \mathrm{C}$ and 2000 bars in the presence of quartz. Am. J. Sci. 272: 234-272.

Piwinskii, A. J. (1968) Experimental studies of igneous rock series, Central Sierra Nevada batholith, California. J. Geol. 76: 548-570.

Powell, M. and Powell, R. (1974) An olivine-clinopyroxene geothermometer. Contr. Mineral. Petrol. 48: 249-263.

Roeder, P. L. (1974) Activity of iron and olivine solubility in basaltic liquids. Earth Planét. Sci. Lett. 24: $397-410$.

- and Emslie, R. F. (1970) Olivine-liquid equilibria. Contr. Mineral. Petrol. 29: 275-289.

Seward, T. M. (1971) The distribution of transition elements in the system CaMgSi $\mathrm{O}_{6}-\mathrm{Na}_{2} \mathrm{Si}_{2} \mathrm{O}_{5}-$ $\mathrm{H}_{2} \mathrm{O}$ at 1000 bars pressure. Chem. Geol. 7: 7395.

Shibata, T. (1976) Phenocryst-bulk rock composition relations of abyssal tholeites and their petrogenetic significance. Geochim. Cosmochim. Acta 40: 1407-1417.

Sigvaldason, G. E. (1974) Basalts from the centre of the assumed Icelandic mantle plume. J. Petrol. 15: 497-524.

Sun, C-O., Williams, R. J. and Sun, S-S. (1974) Distribution coefficients of $\mathrm{Eu}$ and $\mathrm{Sr}$ for 


\section{Heikki Mäkipää}

plagioclase-liquid and clinopyroxene-liquid equilibria in oceanic ridge basalts: an experimental study. Geochim. Cosmochim. Acta 38: $1415-1433$.

Tilley, C. E., Yoder, H. S. Jr. and Schairer, J. F. (1964) New relations on melting of basalts. Carnegie Inst. Washington Yearb. 63: 92-97.

Walker, D., Kirkpatrick, R. J., Longhi, J. and Hays, J. F. (1976) Crystallization history of lunar picritic basalt sample 12002: Phase equilibria and cooling-rate studies. Geol. Soc. Am. Bull. 87: 646-656.
Watson, B. E. (1977) Partitioning of managanese between forsterite and silicate liquid. Geochim. Cosmochim. Acta 41: 1363-1374.

Wood, B. J. (1976) An olivine-clinopyroxene geothermometer. A discussion. Contr. Mineral. Petrol. 56: 297-304.

Yoder, H. S. Jr., Stewart, D. B. and Smith, J. R. (1957) Ternary feldspar. Carnegie Inst. Washington Yearb. 56: 206-214.

Yoder, H. S. Jr. and Tilley, C. E. (1962) Origin of basalt magmas. J. Petrol. 3: 346-532.

Manuscript received, May 15, 1978 